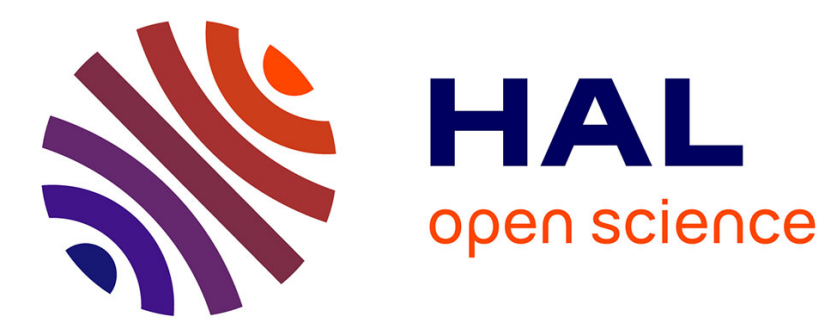

\title{
Undercutting in argumentation systems
}

\author{
Leila Amgoud, Farid Nouioua
}

\section{To cite this version:}

Leila Amgoud, Farid Nouioua. Undercutting in argumentation systems. International Conference on Scalable Uncertainty Management (SUM 2015), Sep 2015, Quebec, Canada. pp. 267-281. hal01592025

\section{HAL Id: hal-01592025 \\ https://hal.science/hal-01592025}

Submitted on 22 Sep 2017

HAL is a multi-disciplinary open access archive for the deposit and dissemination of scientific research documents, whether they are published or not. The documents may come from teaching and research institutions in France or abroad, or from public or private research centers.
L'archive ouverte pluridisciplinaire HAL, est destinée au dépôt et à la diffusion de documents scientifiques de niveau recherche, publiés ou non, émanant des établissements d'enseignement et de recherche français ou étrangers, des laboratoires publics ou privés. 


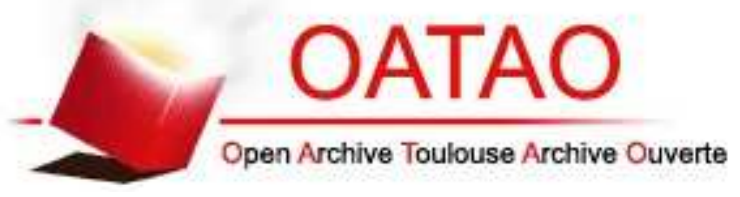

\section{Open Archive TOULOUSE Archive Ouverte (OATAO)}

OATAO is an open access repository that collects the work of Toulouse researchers and makes it freely available over the web where possible.

This is an author-deposited version published in : http://oatao.univ-toulouse.fr/ Eprints ID : 16846

The contribution was presented at SUM 2015 :

http://www.fernuni-hagen.de/wbs/sum2015/

To cite this version : Amgoud, Leila and Nouioua, Farid Undercutting in argumentation systems. (2015) In: International Conference on Scalable Uncertainty Management (SUM 2015), 16 September 2015 - 18 September 2015 (Quebec, Canada).

Any correspondence concerning this service should be sent to the repository administrator: staff-oatao@listes-diff.inp-toulouse.fr 


\title{
Undercutting in argumentation systems
}

\author{
Leila Amgoud $^{1}$ and Farid Nouioua ${ }^{2}$ \\ ${ }^{1}$ IRIT - CNRS, France \\ ${ }^{2}$ LSIS - Aix-Marseille University, France \\ ${ }^{1}$ amgoud@irit.fr, 2 farid.nouioua@lsis.org
}

\begin{abstract}
Rule-based argumentation systems are developed for reasoning about defeasible information. They take as input a theory made of a set of strict rules, which encode strict information, and a set of defeasible rules which describe general behaviour with exceptional cases. They build arguments by chaining such rules, define attacks between them, use a semantics for evaluating the arguments, and finally identify the plausible conclusions that follow from the rules.

One of the main attack relations of such systems is the so-called undercutting which blocks the application of defeasible rules in some contexts. In this paper, we show that this relation is powerful enough to capture alone all the different conflicts in a theory. We present the first argumentation system that uses only undercutting and fully characterize both its extensions and its plausible conclusions under various acceptability semantics.
\end{abstract}

Keywords: Rule-based argumentation, Undercutting, Acceptability semantics.

\section{Introduction}

Rule-based argumentation systems are developed for reasoning about defeasible information. As a major feature, they take as input a theory made of a set of facts, a set of strict rules, which encode strict information, and a set of defeasible rules which describe general behaviour with exceptional cases. They build arguments by chaining such rules, define attacks between them, use a semantics for evaluating the arguments, and finally identify the plausible conclusions that follow from the rules. Examples of such systems are ASPIC [2], its extended version ASPIC+ [14], Delp [8] and the system developed in [11]. Some of these systems satisfy the rationality postulates proposed in [3]. However, the plausible conclusions of any of these systems have never been characterized.

Besides that, systems like Delp use rebuttal as attack relation between arguments. Rebuttal captures the fact that the conclusions of two arguments are inconsistent. Systems like ASPIC [2] and Pollock's system [13] use, in addition to rebuttal, undercut which blocks the application of defeasible rules in particular contexts. Let us illustrate this relation by an example borrowed from [13]. Consider the following argument $a$ :

"The object is red (or) because it looks red $(l r)$ ".

This argument uses of the defeasible rule $l r \Rightarrow$ or (meaning that generally, if an object looks red, then it is red). Assume now another argument $b$ which states the following: 
"The rule $l r \Rightarrow$ or cannot be applied because the object is illuminated by a red light”.

The argument $b$ undercuts $a$ and the conclusion (or) of $a$ is not drawn from the theory. Undercut deals with the exceptions of defeasible rules. Indeed, every exception of a defeasible rule gives birth to an attack from any argument supporting the exception toward any argument using the rule. In the example, being illuminated by a red light is a specific case where the rule $l r \Rightarrow$ or cannot be applied.

In this paper, we argue that undercut can do more than dealing with exceptions of defeasible rules. It can also perfectly play the role of rebuttal, and deal thus with inconsistency. The basic idea is the following: if a theory contains a defeasible rule $x \Rightarrow y$ and $\neg y$ follows from the same theory, then the rule should be blocked. We propose the first rule-based argumentation system that uses undercutting as its single attack relation. We show that it satisfies the rationality postulates discussed in [3] under naive, stable and preferred semantics. From a conceptual point of view, this system is much simpler than existing ones that combine rebuttal and undercut. Indeed, the latter require different variants of rebuttal for satisfying the basic postulates under different semantics. Our system satisfies the postulates under all semantics. Moreover, restricted rebut, one of the variants of rebuttal, is based on assumption which may appear not intuitive. Indeed, this relation compares only the rules whose heads are inconsistent, and neglects the remaining structures of the arguments. For instance, it considers that the argument $\left(x_{1}, x_{1} \Rightarrow y_{1}, y_{1} \rightarrow z\right)$ attacks the argument $\left(x_{2}, x_{2} \rightarrow y_{2}, y_{2} \Rightarrow \neg z\right)$ since $z$ follows from a strict rule while $\neg z$ follows from a defeasible one. Note that the converse is not true even if the the first rule of the first argument is defeasible while that of the second argument is strict. In our system, we do not make such assumptions. The second main contribution of the paper consists of providing the first and full characterizations of the extensions as well as the set of plausible conclusions of our system under naive, stable and preferred semantics proposed in [7].

The paper is organized as follows: The next section defines the rule-based system we are interested in. Then, we analyse its properties and characterize its extensions and set of plausible conclusions in subsequent sections.

\section{Rule-based systems}

As in [1], three kinds of information are distinguished: Facts representing factual information like 'Tweety is a bird', strict rules representing strict information like 'Penguins do not fly' and defeasible rules describing general behavior with exceptional cases like 'Birds fly'. In what follows, $\mathcal{L}$ is a set of literals, i.e. atoms or negation of atoms, representing knowledge. The negation of an atom $x$ from $\mathcal{L}$ is denoted $\neg x . \mathcal{L}^{\prime}$ is a set of atoms used for naming rules. The two sets satisfy the constraint $\mathcal{L} \cap \mathcal{L}^{\prime}=\emptyset$. Every rule has a single name and two rules cannot have the same name. Throughout the paper, rules are named $r, r_{1}, r_{2}, \ldots$ The function $\operatorname{Rule}\left(r_{i}\right)$ returns the rule whose name is $r_{i}$.

- Facts are elements of $\mathcal{L}$.

- Defeasible rules are of the form $x_{1}, \ldots, x_{n} \Rightarrow x$ and $x, x_{1} \ldots, x_{n}$ are literals in $\mathcal{L}$. 
- Strict rules are of the form $x_{1}, \ldots, x_{n} \rightarrow x$ where $x_{1}, \ldots, x_{n}$ are literals of $\mathcal{L}$ and

$$
\left\{\begin{array}{l}
x \in \mathcal{L} \text { or } \\
x \in \mathcal{L}^{\prime} \text { and } \operatorname{Rule}(x) \text { is defeasible. }
\end{array}\right.
$$

Note that the names of rules cannot appear in bodies of (strict or defeasible) rules. This means that it is not possible to represent information of the form "if rule $r$ is applied (or is blocked), then $y$ holds". Moreover, strict rules cannot be blocked. By default, any defeasible rule can be applied, unless explicitly mentioned in the language by strict rules $x_{1}, \ldots, x_{n} \rightarrow x$ with $x \in \mathcal{L}^{\prime}$. Such a rule is read as follows: If $x_{1}, \ldots, x_{n}$ hold, then the defeasible rule $x$ is always not applicable.

Definition 1 (Theory). $A$ theory is a triple $\mathcal{T}=(\mathcal{F}, \mathcal{S}, \mathcal{D})$ where $\mathcal{F} \subseteq \mathcal{L}$ is a set of facts and $\mathcal{S} \subseteq \mathcal{L}^{\prime}$ (respectively $\mathcal{D} \subseteq \mathcal{L}^{\prime}$ ) is a set of strict (respectively defeasible) rules.

Notations: For each rule $x_{1}, \ldots, x_{n} \rightarrow x$ (as well as $x_{1}, \ldots, x_{n} \Rightarrow x$ ) whose name is $r$, the head of the rule is $\operatorname{Head}(r)=x$ and the body of the rule is $\operatorname{Body}(r)=\left\{x_{1}, \ldots, x_{n}\right\}$. Let $\mathcal{T}=(\mathcal{F}, \mathcal{S}, \mathcal{D})$ and $\mathcal{T}^{\prime}=\left(\mathcal{F}^{\prime}, \mathcal{S}^{\prime}, \mathcal{D}^{\prime}\right)$ be two theories. We say that $\mathcal{T}$ is a subtheory of $\mathcal{T}^{\prime}$, written $\mathcal{T} \sqsubseteq \mathcal{T}^{\prime}$, iff $\mathcal{F} \subseteq \mathcal{F}^{\prime}$ and $\mathcal{S} \subseteq \mathcal{S}^{\prime}$ and $\mathcal{D} \subseteq \mathcal{D}^{\prime}$. The relation $\sqsubset$ is the strict version of $\sqsubseteq$ (i.e., it is the case that at least one of the three inclusions is strict). Finally, $\operatorname{Def} \mathbf{s}(\mathcal{T})=\mathcal{D}$.

Let us now show how new information is produced from a given theory. This is generally the case when (strict and/or defeasible) rules are fired in a derivation schema.

Definition 2 (Derivation schema). Let $\mathcal{T}=(\mathcal{F}, \mathcal{S}, \mathcal{D})$ be a theory and $x \in \mathcal{L} \cup \mathcal{L}^{\prime}$. A derivation schema for $x$ from $\mathcal{T}$ is a finite sequence $d=\left\langle\left(x_{1}, r_{1}\right), \ldots,\left(x_{n}, r_{n}\right)\right\rangle$ s.t.

- $x_{n}=x$

- for $i=1 \ldots n$,

- $x_{i} \in \mathcal{F}$ and $r_{i}=\emptyset$, or

- $r_{i} \in \mathcal{S} \cup \mathcal{D}$ and $\operatorname{Head}\left(r_{i}\right)=x_{i}$ and $\operatorname{Body}\left(r_{i}\right) \subseteq\left\{x_{1}, . ., x_{i-1}\right\}$

$\operatorname{Seq}(d)=\left\{x_{1}, \ldots, x_{n}\right\}$.

$\operatorname{Facts}(d)=\left\{x_{i} \mid i \in\{1, \ldots, n\}, r_{i}=\emptyset\right\}$.

$\operatorname{Strict}(d)=\left\{r_{i} \mid i \in\{1, \ldots, n\}, r_{i} \in \mathcal{S}\right\}$.

$\operatorname{Def}(d)=\left\{r_{i} \mid i \in\{1, \ldots, n\}, r_{i} \in \mathcal{D}\right\}$.

$\mathrm{CN}(\mathcal{T})$ denotes the set of all literals that have a derivation schema from $\mathcal{T}$.

It is clear from the definition that $\mathrm{CN}$ is monotonic.

Example 1 Let $\mathcal{T}_{1}=\left(\mathcal{F}_{1}, \mathcal{S}_{1}, \mathcal{D}_{1}\right)$ be a theory such that $\mathcal{F}_{1}=\{p, b\}, \mathcal{S}_{1}=\left\{\left(r_{1}\right) p \rightarrow\right.$ $\neg f\}$ and $\mathcal{D}_{1}=\left\{\left(r_{2}\right) b \Rightarrow f\right\}$. From $\mathcal{T}_{1}$, we have the following minimal derivations:

- $d_{1}=\langle(p, \emptyset)\rangle$

- $d_{2}=\langle(b, \emptyset)\rangle$

- $d_{3}=\left\langle(p, \emptyset),\left(\neg f, r_{1}\right)\right\rangle$

- $d_{4}=\left\langle(b, \emptyset),\left(f, r_{2}\right)\right\rangle$ 
A notion of consistency and another of coherence are associated with this logical language.

Definition 3 (Consistency-Coherence). $A$ set $X \subseteq \mathcal{L}$ is consistent iff $\nexists x, y \in \mathcal{L}$ such that $x=\neg y$. It is inconsistent otherwise.

A theory $\mathcal{T}=(\mathcal{F}, \mathcal{S}, \mathcal{D})$ is consistent iff $\operatorname{CN}(\mathcal{T})$ is consistent. It is coherent iff $\operatorname{CN}(\mathcal{T}) \cap$ $\mathcal{D}=\emptyset$.

The set of strict rules should be closed under transposition. This is required for ensuring the rationality postulates proposed in [3].

Definition 4 (Closure under transposition). Let $\mathcal{S}$ be a set of strict rules. For all rule $r=x_{1}, \ldots, x_{n} \rightarrow x$ with $x \in \mathcal{L}, r^{\prime}$ is a transposition of $r$ iff $r^{\prime}=x_{1}, \ldots, x_{i-1}, \neg x$, $x_{i+1}, \ldots, x_{n} \rightarrow \neg x_{i}$ for some $1 \leq i \leq n$.

We define $C l_{t}(\mathcal{S})$ as the minimal set such that:

- $\mathcal{S} \subseteq C l_{t}(\mathcal{S})$, and

- If $r \in C l_{t}(\mathcal{S})$ and $r^{\prime}$ is a transposition of $r$ then $r^{\prime} \in C l_{t}(\mathcal{S})$.

We say that $\mathcal{S}$ is closed under transposition iff $C l_{t}(\mathcal{S})=\mathcal{S}$.

Throughout the paper, we will consider undercut for capturing all the possible conflicts between arguments. Thus, undercut will be used both for blocking general rules in presence of exceptions of such rules, and also for handling inconsistency. For that purpose, the theory should be closed, that is for each defeasible rule $r$, the theory should contain the strict rule $\neg \operatorname{Head}(r) \rightarrow r$. This closure captures the fact that $\operatorname{Head}(r)$ and $\neg$ Head $(r)$ cannot hold at the same time.

Definition 5 (Closed theory). A theory $\mathcal{T}=(\mathcal{F}, \mathcal{S}, \mathcal{D})$ is closed iff

- $\mathcal{S}$ is closed under transposition, and

- for all $r=x_{1}, \ldots, x_{n} \Rightarrow x \in \mathcal{D}, \neg x \rightarrow r \in \mathcal{S}$.

Example 1 (Cont) The closed version of $\mathcal{T}_{1}$ is $\mathcal{T}_{1}^{\prime}=\left(\mathcal{F}_{1}, \mathcal{S}_{1}^{\prime}, \mathcal{D}_{1}\right)$ such that $\mathcal{S}_{1}^{\prime}=$ $\left\{\left(r_{1}\right) p \rightarrow \neg f,\left(r_{3}\right) f \rightarrow \neg p,\left(r_{4}\right) \neg f \rightarrow r_{2}\right\}$.

The backbone of an argumentation system is naturally the notion of arguments. They are built from a closed theory using the notion of derivation schema as follows.

Definition 6 (Argument). Let $\mathcal{T}=(\mathcal{F}, \mathcal{S}, \mathcal{D})$ be a closed theory. An argument defined from $\mathcal{T}$ is a pair $(d, x)$ s.t.

- $x \in \mathcal{L} \cup \mathcal{L}^{\prime}$

- $d$ is a derivation schema for $x$ from $\mathcal{T}$

- $\nexists \mathcal{T}^{\prime} \sqsubset(\operatorname{Facts}(d), \operatorname{Strict}(d), \operatorname{Def}(d))$ s.t. $x \in \operatorname{CN}\left(\mathcal{T}^{\prime}\right)$

An argument $(d, x)$ is strict iff $\operatorname{Def}(d)=\emptyset$. 
Unlike ASPIC and ASPIC+ systems, arguments are minimal in our system. An argument may have several sub-parts, each of which is called sub-argument.

Definition 7 (Sub-argument). An argument $(d, x)$ is a sub-argument of $\left(d^{\prime}, x^{\prime}\right)$ iff $(\operatorname{Facts}(d), \operatorname{Strict}(d), \operatorname{Def}(d)) \sqsubseteq\left(\operatorname{Facts}\left(d^{\prime}\right), \operatorname{Strict}\left(d^{\prime}\right), \operatorname{Def}\left(d^{\prime}\right)\right)$.

Notations: $\operatorname{Arg}(\mathcal{T})$ denotes the set of all arguments built from theory $\mathcal{T}$ in the sense of Def. 6. If $a=(d, x)$ is an argument, $\operatorname{Conc}(a)=x$ and $\operatorname{Sub}(a)$ is the set of all its sub-arguments. For a set $\mathcal{E}$ of arguments, $\operatorname{Concs}(\mathcal{E})=\{x \mid(d, x) \in \mathcal{E}\}$ and $\operatorname{Th}(\mathcal{E})$ is a theory such that:

$$
\operatorname{Th}(\mathcal{E})=\left(\bigcup_{(d, x) \in \mathcal{E}} \operatorname{Facts}(d), \bigcup_{(d, x) \in \mathcal{E}} \operatorname{Strict}(d), \bigcup_{(d, x) \in \mathcal{E}} \operatorname{Def}(d)\right) .
$$

The undercutting relation is defined as follows:

Definition 8 (Undercutting). Let $\mathcal{T}=(\mathcal{F}, \mathcal{S}, \mathcal{D})$ be a closed theory and $(d, x),\left(d^{\prime}, x^{\prime}\right) \in$ $\operatorname{Arg}(\mathcal{T}) .(d, x)$ undercuts $\left(d^{\prime}, x^{\prime}\right)$, denoted by $(d, x) \mathcal{R}_{u}\left(d^{\prime}, x^{\prime}\right)$, iff $x \in \operatorname{Def}\left(d^{\prime}\right)$.

Let us illustrate this relation by some examples.

Example 1 (Cont) The set $\operatorname{Arg}\left(\mathcal{T}_{1}^{\prime}\right)$ contains:

- $a_{1}:(\langle(b, \emptyset)\rangle, b)$ $a_{2}:(\langle(p, \emptyset)\rangle, p)$

- $a_{3}:\left(\left\langle(p, \emptyset),\left(\neg f, r_{1}\right)\right\rangle, \neg f\right)$

- $a_{4}:\left(\left\langle(p, \emptyset),\left(\neg f, r_{1}\right),\left(r_{2}, r_{4}\right)\right\rangle, r_{2}\right)$

- $a_{5}:\left(\left\langle(b, \emptyset),\left(f, r_{2}\right)\right\rangle, f\right)$

- $a_{6}:\left(\left\langle(b, \emptyset),\left(f, r_{2}\right),\left(\neg p, r_{3}\right)\right\rangle, \neg p\right)$

$a_{4}$ undercuts $a_{5}, a_{6}$ since $r_{2} \in \operatorname{Def}\left(d_{5}\right), r_{2} \in \operatorname{Def}\left(d_{6}\right)$.

Strict arguments cannot be attacked using this relation.

Proposition 1. Let $\mathcal{T}=(\mathcal{F}, \mathcal{S}, \mathcal{D})$ be a theory. For all argument a $\in \operatorname{Arg}((\mathcal{F}, \mathcal{S}, \emptyset))$, $\nexists b \in \operatorname{Arg}(\mathcal{T})$ s.t. $b \mathcal{R}_{u} a$.

Throughout the paper, we study the following rule-based argumentation system.

Definition 9 (Argumentation system). An argumentation system (AS) defined over a closed theory $\mathcal{T}=(\mathcal{F}, \mathcal{S}, \mathcal{D})$ is a pair $\mathcal{H}=\left(\operatorname{Arg}(\mathcal{T}), \mathcal{R}_{u}\right)$ where $\mathcal{R}_{u} \subseteq \operatorname{Arg}(\mathcal{T}) \times$ $\operatorname{Arg}(\mathcal{T})$.

Arguments are evaluated using extension-based semantics [7]. These semantics are based on two key notions:

- Conflict-freeness: A set $\mathcal{E}$ of arguments is conflict-free iff $\nexists a, b \in \mathcal{E}$ s.t. $a \mathcal{R}_{u} b$.

- Defence: A set $\mathcal{E}$ of arguments defends an argument $a$ iff for all argument $b$ s.t. $b \mathcal{R}_{u} a, \exists c \in \mathcal{E}$ s.t. $c \mathcal{R}_{u} b$.

Definition 10 (Semantics). Let $\mathcal{H}=\left(\operatorname{Arg}(\mathcal{T}), \mathcal{R}_{u}\right)$ and $\mathcal{E} \subseteq \operatorname{Arg}(\mathcal{T})$. 
- $\mathcal{E}$ is a naive extension iff it is a maximal (w.r.t. set $\subseteq$ ) conflict-free set.

- $\mathcal{E}$ is a preferred extension iff it is a maximal (w.r.t. set $\subseteq$ ) conflict-free set which defends all its elements.

- $\mathcal{E}$ is a stable extension iff $\mathcal{E}$ is conflict-free and $\forall a \in \operatorname{Arg}(\mathcal{T}) \backslash \mathcal{E}, \exists b \in \mathcal{E}$ such that $b \mathcal{R}_{u} a$.

Notations: $\operatorname{Ext}_{x}(\mathcal{H})$ denotes the set of all extensions of system $\mathcal{H}$ under semantics $x$ where $x \in\{n, p, s\}, n$ (resp. $p, s$ ) stands for naive (resp. preferred, stable). When we do not need to refer to a particular semantics, we write $\operatorname{Ext}(\mathcal{H})$.

The extensions of a system are used for defining the plausible conclusions to be drawn from the theory over which the system is built. A literal is a plausible conclusion of a system iff it is a common conclusion to all the extensions.

Definition 11 (Plausible conclusions). The set of plausible conclusions of an argumentation system $\mathcal{H}$ is $\operatorname{Output}(\mathcal{H})=\emptyset$ if $\operatorname{Ext}(\mathcal{H})=\emptyset$ and

$$
\operatorname{Output}(\mathcal{H})=\bigcap_{\mathcal{E}_{i} \in \operatorname{Ext}(\mathcal{H})} \operatorname{Concs}\left(\mathcal{E}_{i}\right) \text { otherwise. }
$$

Example 1 (Cont) The argumentation system $\mathcal{H}_{1}=\left(\operatorname{Arg}\left(\mathcal{T}_{1}^{\prime}\right), \mathcal{R}_{u}\right)$ has a single stable extension which is also preferred: $\mathcal{E}=\left\{a_{1}, a_{2}, a_{3}, a_{4}\right\}$ and $\operatorname{Output}\left(\mathcal{H}_{1}\right)=\left\{p, b, \neg f, r_{2}\right\}$.

\section{Properties of the system}

Let us now analyse the properties of the argumentation system defined in the previous section. We show that it satisfies all the rationality postulates proposed in [3]. Indeed, every extension (under any of the reviewed semantics) contains all the sub-arguments of its arguments. The system is also coherent, that is it is not possible for an extension to use a defeasible rule in one of its arguments, and at the same time to block that rule by another argument. In addition, for preferred and stable semantics, every extension returns a consistent set of conclusions (unless the strict part of the theory is inconsistent) and the set of conclusions of every extension is closed under strict rules (under stable and preferred semantics), that is it is not possible that an extension supports a conclusion $x$ and forgets $y$ if $x \rightarrow y \in \mathcal{S}$.

Theorem 1. Let $\mathcal{H}=\left(\operatorname{Arg}(\mathcal{T}), \mathcal{R}_{u}\right)$ be an argumentation system built over a closed theory $\mathcal{T}=(\mathcal{F}, \mathcal{S}, \mathcal{D})$ s.t. $\operatorname{Ext}(\mathcal{H}) \neq \emptyset$. For all $\mathcal{E} \in \operatorname{Ext}(\mathcal{H})$, the following hold:

- $\operatorname{Th}(\mathcal{E})$ is coherent,

- for all $a \in \mathcal{E}, \operatorname{Sub}(a) \subseteq \mathcal{E}$,

Under stable and preferred semantics, consistency and closure under strict rules are also satisfied.

Theorem 2. Let $\mathcal{H}=\left(\operatorname{Arg}(\mathcal{T}), \mathcal{R}_{u}\right)$ be an argumentation system built over a closed theory $\mathcal{T}=(\mathcal{F}, \mathcal{S}, \mathcal{D})$ s.t. $\operatorname{Ext}_{x}(\mathcal{H}) \neq \emptyset$ with $x \in\{s, p\}$. For all $\mathcal{E} \in \operatorname{Ext}_{x}(\mathcal{H})$, the following hold: 
- Concs $(\mathcal{E})$ is consistent iff $\mathrm{CN}((\mathcal{F}, \mathcal{S}, \emptyset))$ is consistent,

- $\operatorname{Concs}(\mathcal{E})=\operatorname{CN}((\operatorname{Concs}(\mathcal{E}), \mathcal{S}, \emptyset))$,

- Output $(\mathcal{H})=\operatorname{CN}((\operatorname{Output}(\mathcal{H}), \mathcal{S}, \emptyset))$.

The following property follows from the previous theorem.

Corollary 1. Let $\mathcal{H}=\left(\operatorname{Arg}(\mathcal{T}), \mathcal{R}_{u}\right)$ be an argumentation system built over a closed theory $\mathcal{T}=(\mathcal{F}, \mathcal{S}, \mathcal{D})$ s.t. $\operatorname{Ext}_{x}(\mathcal{H}) \neq \emptyset$ with $x \in\{s, p\}$. Output $(\mathcal{H})$ is consistent iff $\operatorname{CN}((\mathcal{F}, \mathcal{S}, \emptyset))$ is consistent,

The previous results show that the outcomes of the argumentation system (its extensions and set of plausible conclusions) satisfy nice properties. However, they do not say anything about the kind of conclusions the system draws from a theory. We answer this question in the next section.

\section{The outputs of the system}

This section provides formal characterizations of the outputs of the system under the three reviewed semantics. For each semantics, we characterize the extensions in terms of sub-theories of the theory over which the system is built, delimit the number of extensions, and fully characterize the set of plausible conclusions.

\subsection{Naive semantics}

A sub-theory that corresponds to a naive extension is called option and is defined as follows:

Definition 12 (Option). An option of a closed theory $\mathcal{T}=(\mathcal{F}, \mathcal{S}, \mathcal{D})$ is a sub-theory $\left(\mathcal{F}^{\prime}, \mathcal{S}^{\prime}, \mathcal{D}^{\prime}\right)$ such that

- $\mathcal{F}^{\prime}=\mathcal{F}, \mathcal{S}^{\prime} \subseteq \mathcal{S}$ and $\mathcal{D}^{\prime} \subseteq \mathcal{D}$

- $\left(\mathcal{F}^{\prime}, \mathcal{S}^{\prime}, \mathcal{D}^{\prime}\right)$ is coherent

- $\forall r \in \mathcal{S}^{\prime} \cup \mathcal{D}^{\prime}, \operatorname{Body}(r) \subseteq \operatorname{CN}\left(\left(\mathcal{F}^{\prime}, \mathcal{S}^{\prime}, \mathcal{D}^{\prime}\right)\right)$

- $\nexists \mathcal{S}^{\prime \prime}, \mathcal{D}^{\prime \prime}$ such that $\left(\mathcal{F}^{\prime}, \mathcal{S}^{\prime}, \mathcal{D}^{\prime}\right) \sqsubset\left(\mathcal{F}^{\prime}, \mathcal{S}^{\prime \prime}, \mathcal{D}^{\prime \prime}\right)$ and $\left(\mathcal{F}^{\prime}, \mathcal{S}^{\prime \prime}, \mathcal{D}^{\prime \prime}\right)$ satisfies the previous conditions.

$\operatorname{Opt}(\mathcal{T})$ denotes the set of options of the closed theory $\mathcal{T}$.

Thus, an option is obtained by taking all the facts and a maximal (w.r.t set inclusion) subset of (strict and defeasible) rules so that the sub-theory remains coherent and all the added rules are applicable. Notice that no priority is given to strict rules over defeasible ones. This is explained by the fact that naive semantics does not distinguish between attackers and attacked arguments. 
Example 2 Consider the closed theory $\mathcal{T}_{3}=\left(\mathcal{F}_{3}, \mathcal{S}_{3}, \mathcal{D}_{3}\right)$ :

$\mathcal{F}_{3}\left\{\begin{array}{l}x \\ y\end{array} \quad \mathcal{S}_{3}\left\{\begin{array}{ll}t \rightarrow r_{2} & \left(r_{4}\right) \\ u \rightarrow r_{1} & \left(r_{5}\right) \\ s \rightarrow r_{3} & \left(r_{6}\right)\end{array} \quad \mathcal{D}_{3} \begin{cases}x \Rightarrow t & \left(r_{1}\right) \\ y \Rightarrow u & \left(r_{2}\right) \\ t \Rightarrow s & \left(r_{3}\right)\end{cases}\right.\right.$

The theory $\mathcal{T}_{3}$ has three options:

- $\mathcal{O}_{1}=\left(\mathcal{F}_{3}, \emptyset,\left\{r_{1}, r_{2}, r_{3}\right\}\right)$

$\operatorname{CN}\left(\mathcal{O}_{1}\right)=\{x, y, t, u, s\}$

- $\mathcal{O}_{2}=\left(\mathcal{F}_{3},\left\{r_{4}\right\},\left\{r_{1}, r_{3}\right\}\right)$

$\mathrm{CN}\left(\mathcal{O}_{2}\right)=\left\{x, y, t, s, r_{2}\right\}$

- $\mathcal{O}_{3}=\left(\mathcal{F}_{3},\left\{r_{5}\right\},\left\{r_{2}\right\}\right)$

$\mathrm{CN}\left(\mathcal{O}_{3}\right)=\left\{x, y, u, r_{1}\right\}$

Let us now establish the relationship between naive extensions of an argumentation system and the options of the closed theory over which it is built. Each naive extension returns one option and two naive extensions cannot return the same option.

Theorem 3. Let $\mathcal{H}=\left(\operatorname{Arg}(\mathcal{T}), \mathcal{R}_{u}\right)$ be an argumentation system built over a closed theory $\mathcal{T}$.

- For all $\mathcal{E} \in \operatorname{Ext}_{n}(\mathcal{H})$, there exists a single option $\mathcal{O} \in \operatorname{Opt}(\mathcal{T})$ such that $\operatorname{Th}(\mathcal{E})=$ $\mathcal{O}$ and $\operatorname{Concs}(\mathcal{E})=\operatorname{CN}(\mathcal{O})$. We put: $\operatorname{Option}(\mathcal{E}) \stackrel{\text { def }}{=} \mathcal{O}$.

- For all $\mathcal{E}, \mathcal{E}^{\prime} \in \operatorname{Ext}_{n}(\mathcal{H})$, if Option $(\mathcal{E})=\operatorname{Option}\left(\mathcal{E}^{\prime}\right)$ then $\mathcal{E}=\mathcal{E}^{\prime}$.

- For all $\mathcal{E} \in \operatorname{Ext}_{n}(\mathcal{H}), \mathcal{E}=\operatorname{Arg}(\operatorname{Option}(\mathcal{E}))$.

The following theorem shows that inversely, each option leads to one naive extension and two different options do not return the same naive extension.

Theorem 4. Let $\mathcal{H}=\left(\operatorname{Arg}(\mathcal{T}), \mathcal{R}_{u}\right)$ be an argumentation system built over a closed theory $\mathcal{T}$.

- For all $\mathcal{O} \in \operatorname{Opt}(\mathcal{T}), \operatorname{Arg}(\mathcal{O}) \in \operatorname{Ext}_{n}(\mathcal{H})$.

- For all $\mathcal{O} \in \operatorname{Opt}(\mathcal{T}), \mathcal{O}=\operatorname{Option}(\operatorname{Arg}(\mathcal{O}))$.

- For all $\mathcal{O}_{1}, \mathcal{O}_{2} \in \operatorname{Opt}(\mathcal{T})$, if $\operatorname{Arg}\left(\mathcal{O}_{1}\right)=\operatorname{Arg}\left(\mathcal{O}_{2}\right), \mathcal{O}_{1}=\mathcal{O}_{2}$.

Example 2 (Cont) The arguments built from $\mathcal{T}_{3}$ are summarized below.

- $a_{1}:(\langle(x, \emptyset)\rangle, x)$

- $a_{2}:(\langle(y, \emptyset)\rangle, y)$

- $a_{3}:\left(\left\langle(x, \emptyset),\left(t, r_{1}\right)\right\rangle, t\right)$

- $a_{4}:\left(\left\langle(x, \emptyset),\left(t, r_{1}\right),\left(r_{2}, r_{4}\right)\right\rangle, r_{2}\right)$

- $a_{5}:\left(\left\langle(y, \emptyset),\left(u, r_{2}\right)\right\rangle, u\right)$

- $a_{6}:\left(\left\langle(y, \emptyset),\left(u, r_{2}\right),\left(r_{1}, r_{5}\right)\right\rangle, r_{1}\right)$

- $a_{7}:\left(\left\langle(x, \emptyset),\left(t, r_{1}\right),\left(s, r_{3}\right)\right\rangle, s\right)$

- $a_{8}:\left(\left\langle(x, \emptyset),\left(t, r_{1}\right),\left(s, r_{3}\right),\left(r_{3}, r_{6}\right)\right\rangle, r_{3}\right)$ 


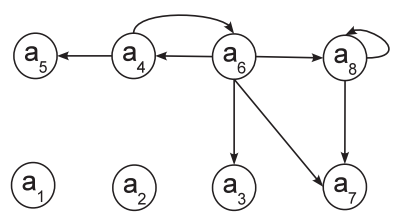

Fig. 1. Graph of attacks built from the theory $\mathcal{T}_{3}$

The graph of attacks is depicted in the figure 1 below:

The argumentation system $\mathcal{H}_{3}=\left(\operatorname{Arg}\left(\mathcal{T}_{3}\right), \mathcal{R}_{u}\right)$ has three naive extensions $\mathcal{E}_{1}=$ $\left\{a_{1}, a_{2}, a_{3}, a_{5}, a_{7}\right\}, \mathcal{E}_{2}=\left\{a_{1}, a_{2}, a_{3}, a_{4}, a_{7}\right\}$ and $\mathcal{E}_{3}=\left\{a_{1}, a_{2}, a_{5}, a_{6}\right\}$ which capture the options $\mathcal{O}_{1}, \mathcal{O}_{2}$ and $\mathcal{O}_{3}$ respectively. Indeed, $\operatorname{Th}\left(\mathcal{E}_{1}\right)=\mathcal{O}_{1}$ (resp. $\operatorname{Th}\left(\mathcal{E}_{2}\right)=\mathcal{O}_{2}$, $\left.\operatorname{Th}\left(\mathcal{E}_{3}\right)=\mathcal{O}_{3}\right)$ and $\operatorname{Concs}\left(\mathcal{E}_{1}\right)=\operatorname{CN}\left(\mathcal{O}_{1}\right)\left(\right.$ resp. $\operatorname{Concs}\left(\mathcal{E}_{2}\right)=\operatorname{CN}\left(\mathcal{O}_{2}\right)$, $\operatorname{Concs}\left(\mathcal{E}_{3}\right)=$ $\mathrm{CN}\left(\mathcal{O}_{3}\right)$ ).

From the previous correspondence, the number of naive extensions is delimited as follows:

Corollary 2. Let $\mathcal{H}=\left(\operatorname{Arg}(\mathcal{T}), \mathcal{R}_{u}\right)$ be an argumentation system. It holds that $\left|\operatorname{Ext}_{n}(\mathcal{H})\right|=$ $|\operatorname{Opt}(\mathcal{T})|$.

The plausible conclusions of the argumentation system under naive semantics are exactly the literals that follow from all the options of the theory over which the system is built.

Corollary 3. Let $\mathcal{H}=\left(\operatorname{Arg}(\mathcal{T}), \mathcal{R}_{u}\right)$ be an argumentation system. Output $(\mathcal{H})=$ $\bigcap_{\mathcal{O} \in \mathrm{Opt}(\mathcal{T})} \operatorname{CN}(\mathcal{O})$.

Example 2 (Cont) Under naive semantics, Output $(\mathcal{H})=\operatorname{CN}\left(\mathcal{O}_{1}\right) \cap \operatorname{CN}\left(\mathcal{O}_{2}\right) \cap \operatorname{CN}\left(\mathcal{O}_{3}\right)=$ $\{x, y\}$.

\subsection{Stable semantics}

The sub-theories of a closed theory that capture stable extensions are called strong options and are defined as follows:

Definition 13 (Strong Option). A strong option of a closed theory $\mathcal{T}=(\mathcal{F}, \mathcal{S}, \mathcal{D})$ is a sub-theory $\left(\mathcal{F}^{\prime}, \mathcal{S}^{\prime}, \mathcal{D}^{\prime}\right)$ s.t.

- $\mathcal{F}^{\prime}=\mathcal{F}, \mathcal{S}^{\prime}=\mathcal{S}$ and $\mathcal{D}^{\prime} \subseteq \mathcal{D}$

- $\left(\mathcal{F}^{\prime}, \mathcal{S}^{\prime}, \mathcal{D}^{\prime}\right)$ is coherent

- $\forall r \in \mathcal{D}^{\prime}, \operatorname{Body}(r) \subseteq \mathrm{CN}\left(\left(\mathcal{F}^{\prime}, \mathcal{S}^{\prime}, \mathcal{D}^{\prime}\right)\right)$

- $\forall r \notin \mathcal{D}^{\prime}$ we have: either $r \in \operatorname{CN}\left(\mathcal{F}^{\prime}, \mathcal{S}^{\prime}, \mathcal{D}^{\prime}\right)$ or $\exists x \in \operatorname{Body}(r)$ such that $x \notin$ $\mathrm{CN}\left(\mathcal{F}^{\prime}, \mathcal{S}^{\prime}, \mathcal{D}^{\prime}\right)$ 
$\operatorname{SOpt}(\mathcal{T})$ denotes the set of strong options of theory $\mathcal{T}$.

In a strong option $\mathcal{O}=\left(\mathcal{F}, \mathcal{S}, \mathcal{D}^{\prime}\right)$ it is not necessary that all the strict rules of $\mathcal{S}$ are applicable. Let $S^{\prime \prime}$ be the subset of strict rules that are applicable in $\mathcal{O}$, i.e., $\mathcal{S}^{\prime \prime}=\{r \in \mathcal{S} \mid \operatorname{Body}(r) \subseteq \operatorname{CN}(\mathcal{O})\}$. Then, the sub-theory $\mathcal{O}^{\prime}=\left(\mathcal{F}, \mathcal{S}^{\prime \prime}, \mathcal{D}^{\prime}\right)$ is an option of $\mathcal{T}$ which clearly has the same conclusions as $\mathcal{O}$ (i.e., $\operatorname{CN}(\mathcal{O})=\operatorname{CN}\left(\mathcal{O}^{\prime}\right)$ ). In addition, every strict (resp. defeasible) rule $r$ which is kept outside $\mathcal{O}^{\prime}$ is not applicable (resp. is not applicable or is such that $r \in \operatorname{CN}\left(\mathcal{O}^{\prime}\right)$ ). This latter constraint does not hold necessarily for every option. Accordingly, every strong option corresponds to a single option but the converse is not true.

Thus, in addition to an "internal condition" (coherence) satisfied by both options and strong options, the latter require an additional "external condition" which consists of justifying each rule kept outside. Notice, that this idea is not new in non-monotonic reasoning. We find it namely in the distinction between Reiter's extensions [15] and Lukaszewicz's extensions [12] in default logic as well as between answer sets [10] and $\iota$-answer sets [9] in logic programming. Let us illustrate strong options and their relationship with options in our running example.

Example 2 (Cont) The theory $\mathcal{T}_{3}$ has one strong option $\mathcal{O}=\left(\mathcal{F}_{3}, \mathcal{S}_{3},\left\{r_{2}\right\}\right)$. Note that the only strict rule in $\mathcal{S}_{3}$ which is applicable for $\mathcal{O}$ is $r_{5}$. If we discard from $\mathcal{O}$ the remaining non-applicable strict rules, we get exactly the option $\mathcal{O}_{3}\left(\mathrm{CN}(\mathcal{O})=\mathrm{CN}\left(\mathcal{O}_{3}\right)\right)$. Note also that each rule which is not included in $\mathcal{O}_{3}$ is justified. Namely, the strict rules $r_{4}$ and $r_{6}$ are note applicable $\left(t \in \operatorname{Body}\left(r_{4}\right), t \notin \operatorname{CN}\left(\mathcal{O}_{3}\right), s \in \operatorname{Body}\left(r_{6}\right)\right.$, and $\left.s \notin \mathrm{CN}\left(\mathcal{O}_{3}\right)\right)$; the defeasible rule $r_{1}$ is such that $r_{1} \in \operatorname{CN}\left(\mathcal{O}_{3}\right)$ and the defeasible rule $r_{3}$ is not applicable $\left(t \in \operatorname{Body}\left(r_{3}\right)\right.$ and $\left.t \notin \operatorname{CN}\left(\mathcal{O}_{3}\right)\right)$. So $\mathcal{O}_{3}$ gives rise to a strong option by adding all the non-applicable strict rules. This is not the case for $\mathcal{O}_{1}$ and $\mathcal{O}_{2}$. Indeed, adding the missing strict rules to them leads to incoherent sub-theories.

It is worthy to say that a closed theory may not have strong options. This is not surprising since as we will show, there is a bijection between the set of stable extensions and the set of strong options. Indeed, every stable extension gives birth to a strong option and two stable extensions cannot return the same strong option.

Theorem 5. Let $\mathcal{H}=\left(\operatorname{Arg}(\mathcal{T}), \mathcal{R}_{u}\right)$ be an argumentation system built over a closed theory $\mathcal{T}$ s.t. $\operatorname{Ext}_{s}(\mathcal{H}) \neq \emptyset$.

- For all $\mathcal{E} \in \operatorname{Ext}_{s}(\mathcal{H})$, there exists a single strong option $\mathcal{O} \in \operatorname{SOpt}(\mathcal{T})$ s.t. $\operatorname{Th}(\mathcal{E}) \sqsubseteq \mathcal{O}$ and $\operatorname{Concs}(\mathcal{E})=\operatorname{CN}(\mathcal{O})$. We put $\operatorname{SOption}(\mathcal{E}) \stackrel{\text { def }}{=} \mathcal{O}$.

- For all $\mathcal{E}, \mathcal{E}^{\prime} \in \operatorname{Ext}_{s}(\mathcal{H})$, if $\operatorname{SOption}(\mathcal{E})=\operatorname{SOption}\left(\mathcal{E}^{\prime}\right)$ then $\mathcal{E}=\mathcal{E}^{\prime}$.

- For all $\mathcal{E} \in \operatorname{Ext}_{s}(\mathcal{H}), \mathcal{E}=\operatorname{Arg}(\operatorname{SOption}(\mathcal{E}))$.

Inversely, every strong option leads to one stable extension and two strong options cannot lead the same stable extension.

Theorem 6. Let $\mathcal{H}=\left(\operatorname{Arg}(\mathcal{T}), \mathcal{R}_{u}\right)$ be an argumentation system built over a closed theory $\mathcal{T}$ s.t. $\operatorname{Ext}_{s}(\mathcal{H}) \neq \emptyset$. 
- For all $\mathcal{O} \in \operatorname{SOpt}(\mathcal{T}), \operatorname{Arg}(\mathcal{O}) \in \operatorname{Ext}_{s}(\mathcal{H})$.

- For all $\mathcal{O} \in \operatorname{SOpt}(\mathcal{T}), \mathcal{O}=\operatorname{SOption}(\operatorname{Arg}(\mathcal{O}))$.

- For all $\mathcal{O}_{1}, \mathcal{O}_{2} \in \operatorname{SOpt}(\mathcal{T})$, if $\operatorname{Arg}\left(\mathcal{O}_{1}\right)=\operatorname{Arg}\left(\mathcal{O}_{2}\right)$ then $\mathcal{O}_{1}=\mathcal{O}_{2}$.

Example 2 (Cont) Among the three naive extensions of the argumentation system $\mathcal{H}_{3}$ built from $\mathcal{T}_{3}$, the only stable extension is $\mathcal{E}_{3}$ which captures the strong options $\mathcal{O}$. Indeed, $\operatorname{Th}\left(\mathcal{E}_{3}\right) \sqsubseteq \mathcal{O}$ and $\operatorname{Concs}\left(\mathcal{E}_{3}\right)=\operatorname{CN}(\mathcal{O})$.

We have seen so far that there is a one to one correspondence between naive (resp. stable) extensions and options (resp. strong options). We have also shown that every strong option is a sub-theory of one option. Thus, the number of stable extensions of a rule-based system is delimited as follows.

Corollary 4. Let $\mathcal{H}=\left(\operatorname{Arg}(\mathcal{T}), \mathcal{R}_{u}\right)$ be an argumentation system built over a closed theory $\mathcal{T}$. The following holds: $0 \leq\left|\operatorname{Ext}_{s}(\mathcal{H})\right|=|\operatorname{SOpt}(\mathcal{T})| \leq|\operatorname{Dpt}(\mathcal{T})|$.

Under stable semantics, the plausible conclusions of an argumentation system are the literals that follow from all the strong options of the theory over which the system is built.

Corollary 5. Let $\mathcal{H}=\left(\operatorname{Arg}(\mathcal{T}), \mathcal{R}_{u}\right)$ be an argumentation system built over a closed theory $\mathcal{T}$ s.t. $\operatorname{Ext}_{s}(\mathcal{H}) \neq \emptyset$. Output $(\mathcal{H})=\bigcap_{\mathcal{O} \in \operatorname{Sopt}(\mathcal{T})} \operatorname{CN}(\mathcal{O})$.

Example 2 (Cont) $\mathcal{O}$ is the only strong option of $\mathcal{T}_{3}$. Thus, $\operatorname{Output}(\mathcal{H})=\operatorname{CN}(\mathcal{O})=$ $\left\{x, y, u, r_{1}\right\}$.

Let us summarize: rule-based argumentation systems may not have stable extensions in which case they miss intuitive conclusions like facts. Systems that do have stable extensions return exactly the literals that follow from all the strong options of the closed theory at hand.

\subsection{Preferred semantics}

We show next that the sub-theories that capture preferred extensions are the so-called preferred options.

Definition 14 (Preferred Option). $A$ preferred option of a closed theory $\mathcal{T}=(\mathcal{F}, \mathcal{S}, \mathcal{D})$ is a sub-theory $\left(\mathcal{F}^{\prime}, \mathcal{S}^{\prime}, \mathcal{D}^{\prime}\right)$ s.t.

- $\mathcal{F}^{\prime}=\mathcal{F}, \mathcal{S}^{\prime}=\mathcal{S}$ and $\mathcal{D}^{\prime} \subseteq \mathcal{D}$

- $\left(\mathcal{F}^{\prime}, \mathcal{S}^{\prime}, \mathcal{D}^{\prime}\right)$ is coherent

- $\forall r \in \mathcal{D}^{\prime}, \operatorname{Body}(r) \subseteq \mathrm{CN}\left(\left(\mathcal{F}^{\prime}, \mathcal{S}^{\prime}, \mathcal{D}^{\prime}\right)\right)$

- $\forall \mathcal{D}^{\prime \prime} \subseteq \mathcal{D}$, if $\exists r^{\prime} \in \mathcal{D}^{\prime}$ such that $r^{\prime} \in \operatorname{CN}\left(\mathcal{F}, \mathcal{S}, \mathcal{D}^{\prime \prime}\right)$ then $\exists r^{\prime \prime} \in \mathcal{D}^{\prime \prime}$ such that $r^{\prime \prime} \in \operatorname{CN}\left(\mathcal{F}, \mathcal{S}, \mathcal{D}^{\prime}\right)$

- $\nexists \mathcal{D}^{\prime \prime}$ such that $\mathcal{D}^{\prime} \subset \mathcal{D}^{\prime \prime}$ and $\left(\mathcal{F}^{\prime}, \mathcal{S}^{\prime}, \mathcal{D}^{\prime \prime}\right)$ satisfies the previous conditions. 
$\operatorname{POpt}(\mathcal{T})$ denotes the set of preferred options of theory $\mathcal{T}$.

Preferred options are between options and strong options:

- Every strong option of a theory $\mathcal{T}$ is a preferred option of $\mathcal{T}$. The converse is not true.

- Every preferred option is a sub-part of an option. More precisely, for every preferred option $\mathcal{O}=\left(\mathcal{F}, \mathcal{S}, \mathcal{D}^{\prime}\right)$, if $\mathcal{S}^{\prime \prime}$ is the subset of strict rules that are applicable in $\mathcal{O}$, i.e., $\mathcal{S}^{\prime \prime}=\{r \in \mathcal{S} \mid \operatorname{Body}(r) \subseteq \mathrm{CN}(\mathcal{O})\}$, then there is a unique option $\mathcal{O}^{\prime}$ such that $\mathcal{O}^{\prime \prime}=\left(\mathcal{F}, \mathcal{S}^{\prime \prime}, \mathcal{D}^{\prime}\right) \sqsubseteq \mathcal{O}^{\prime}$ and $\operatorname{CN}(\mathcal{O})=\operatorname{CN}\left(\mathcal{O}^{\prime \prime}\right) \subseteq \mathrm{CN}\left(O^{\prime}\right)$.

Example 2 (Cont) Consider again the closed theory $\mathcal{T}_{3}$. There are three sub-theories that satisfy the four first conditions of definition 14: $\mathcal{O}_{0}=\left(\mathcal{F}_{3}, \mathcal{S}_{3}, \emptyset\right), \mathcal{O} p_{1}=\left(\mathcal{F}_{3}, \mathcal{S}_{3},\left\{r_{2}\right\}\right)$ and $\mathcal{O} p_{2}=\left(\mathcal{F}_{3}, \mathcal{S}_{3},\left\{r_{1}\right\}\right)$. The maximal ones (that satisfy also the last condition of definition 14) are $\mathcal{O} p_{1}$ and $\mathcal{O} p_{2}$. Notice that $\mathcal{O} p_{1}$ is exactly the unique strong option of $\mathcal{T}_{3}$. The other preferred option $\mathcal{O} p_{2}$ captures a sub-part of the option $\mathcal{O}_{2}=$ $\left(\mathcal{F}_{3},\left\{r_{4}\right\},\left\{r_{1}, r_{3}\right\}\right)$. Indeed, by keeping in $\mathcal{O} p_{2}$ only the strict rues that are applicable we obtain: $\mathcal{O} p_{2}^{\prime}=\left(\mathcal{F}_{3},\left\{r_{4}\right\},\left\{r_{1}\right\}\right)$. We have : $\mathcal{O} p_{2}^{\prime} \sqsubseteq \mathcal{O}_{2}$ and $\operatorname{CN}\left(\mathcal{O} p_{2}\right)=\operatorname{CN}\left(\mathcal{O} p_{2}^{\prime}\right) \subseteq$ $\mathrm{CN}\left(\mathcal{O}_{2}\right)$.

Now, we show that every preferred extension leads to a preferred option and two preferred extensions cannot return the same preferred option.

Theorem 7. Let $\mathcal{H}=\left(\operatorname{Arg}(\mathcal{T}), \mathcal{R}_{u}\right)$ be an argumentation system built over a closed theory $\mathcal{T}$.

- For all $\mathcal{E} \in \operatorname{Ext}_{p}(\mathcal{H})$, there exists a single preferred option $\mathcal{O} \in \operatorname{POpt}(\mathcal{T})$ s.t. $\operatorname{Th}(\mathcal{E}) \sqsubseteq \mathcal{O}$ and $\operatorname{Concs}(\mathcal{E})=\operatorname{CN}(\mathcal{O})$. We put: $\operatorname{POption}(\mathcal{E}) \stackrel{\text { def }}{=} \mathcal{O}$.

- For all $\mathcal{E}, \mathcal{E}^{\prime} \in \operatorname{Ext}_{p}(\mathcal{H})$, if $\operatorname{POption}(\mathcal{E})=\operatorname{POption}\left(\mathcal{E}^{\prime}\right)$ then $\mathcal{E}=\mathcal{E}^{\prime}$.

- For all $\mathcal{E} \in \operatorname{Ext}_{p}(\mathcal{H}), \mathcal{E}=\operatorname{Arg}(\operatorname{POption}(\mathcal{E}))$.

Inversely, every preferred option corresponds to a unique preferred extension and two preferred options cannot return the same preferred extension.

Theorem 8. Let $\mathcal{H}=\left(\operatorname{Arg}(\mathcal{T}), \mathcal{R}_{u}\right)$ be an argumentation system built over a closed theory $\mathcal{T}$.

- For all $\mathcal{O} \in \operatorname{POpt}(\mathcal{T}), \operatorname{Arg}(\mathcal{O}) \in \operatorname{Ext}_{p}(\mathcal{H})$.

- For all $\mathcal{O} \in \operatorname{POpt}(\mathcal{T}), \mathcal{O}=\operatorname{POption}(\operatorname{Arg}(\mathcal{O}))$.

- For all $\mathcal{O}_{1}, \mathcal{O}_{2} \in \operatorname{POpt}(\mathcal{T})$, if $\operatorname{Arg}\left(\mathcal{O}_{1}\right)=\operatorname{Arg}\left(\mathcal{O}_{2}\right)$ then $\mathcal{O}_{1}=\mathcal{O}_{2}$.

Example 2 (Cont) The system $\mathcal{H}_{3}$ constructed from $\mathcal{T}_{3}$ has two preferred extensions $: \mathcal{E} p_{1}=\left\{a_{1}, a_{2}, a_{5}, a_{6}\right\}$ and $\mathcal{E} p_{2}=\left\{a_{1}, a_{2}, a_{3}, a_{4}\right\}$. They capture the preferred options $\mathcal{O} p_{1}$ and $\mathcal{O} p_{2}$ respectively. Indeed, $\operatorname{Th}\left(\mathcal{E} p_{1}\right) \sqsubseteq \mathcal{O} p_{1}\left(\right.$ resp. $\left.\operatorname{Th}\left(\mathcal{E} p_{2}\right) \sqsubseteq \mathcal{O} p_{2}\right)$ and $\operatorname{Concs}\left(\mathcal{E} p_{1}\right)=\operatorname{CN}\left(\mathcal{O} p_{1}\right)\left(\right.$ resp. $\left.\operatorname{Concs}\left(\mathcal{E} p_{2}\right)=\operatorname{CN}\left(\mathcal{O} p_{2}\right)\right)$.

The number of preferred extensions of an argumentation system $\mathcal{H}$ is exactly the number of preferred options of the theory over which the system is built. 
Corollary 6. Let $\mathcal{H}=\left(\operatorname{Arg}(\mathcal{T}), \mathcal{R}_{u}\right)$ be an argumentation system built over a closed theory $\mathcal{T}$. It holds that $\left|\operatorname{Ext}_{p}(\mathcal{H})\right|=|\operatorname{POpt}(\mathcal{T})|$.

The plausible conclusions of an argumentation system, under preferred semantics, are the literals that follow from all the preferred options of the theory at hand.

Corollary 7. Let $\mathcal{H}=\left(\operatorname{Arg}(\mathcal{T}), \mathcal{R}_{u}\right)$ be an argumentation system built over a closed theory $\mathcal{T}$. Output $(\mathcal{H})=\bigcap_{\mathcal{O} \in \operatorname{POpt}(\mathcal{T})} \operatorname{CN}(\mathcal{O})$.

Example 2 (Cont) Consider the theory $\mathcal{T}_{3}$. Output $(\mathcal{H})=\operatorname{CN}\left(\mathcal{O} p_{1}\right) \cap \operatorname{CN}\left(\mathcal{O} p_{2}\right)=$ $\{x, y\}$.

\section{Related work}

There are a couple of rule-based argumentation systems in the literature. Some of them like ASPIC and its extended version ASPIC+ are shown to satisfy the rationality postulates defined in [3], namely the consistency and closure under strict rules of their sets of plausible conclusions. While this is testimony to some strength of these formalisms, it does not say anything about the kind of plausible conclusions they draw from a theory. Surprisingly, the outputs of these systems (their extensions and their plausible conclusions) have never been characterized. The authors of those systems provide only examples to show that the outputs are meaningful. This is certainly not sufficient. Our paper is the first that attempts a systematic study of the outcomes of rule-based systems under naive, stable and preferred semantics. There are two notable exceptions. The first work, done in [1], considered a fragment of our logical language and rebuttal as attack relation. Blocking rules was not allowed. Extensions were characterized in terms of sub-theories. However, some sub-theories may not have corresponding extensions. Thus, there is no bijection between the two. Our formalism is thus more general and our characterisations of its outcomes are more accurate since they are one-to-one correspondences. The second work, done in [4], investigated the link between the logic programming semantics and argumentation ones. The theory over which an argumentation system is built is a logic program, that is, only one type of rules is used. Thus, the logical language is very different from ours.

In addition to the characterizations of the system's outcomes, the other main novelty of our paper is the exclusive use of undercut for encoding conflicts between arguments. This relation is used in some existing systems but not alone. It is always coupled with rebuttal which handles inconsistency. In our paper, we have shown that undercut is powerful enough to perfectly fulfil the role of rebuttal. Moreover, the system satisfies all the rationality postulates under any semantics while in ASPIC and ASPIC+, for each semantics, one should use a different definition of rebuttal in order to satisfy the postulates.

Regarding the definition of undercut, there are three proposals in the literature and they are all equivalent. The first definition is the one followed in our paper and in [14]. The idea is to assign a name to every defeasible rule and to allow these names to be in heads of other rules. Unlike in [14], in our paper, names of rules may only be in heads of strict rules. The reason is that undercut shows exceptions of defeasible rules, 
and exceptions are certain information. For instance, in case of penguin, the rule "birds fly" is never applicable. The second definition, given in [13] and followed in [3], uses an objectivation operator which transforms any defeasible rule into a literal. The latter plays the role of the name of the rule in our system. The last definition, proposed in $[5,6]$, extends the logical language by a new form of rules with which one can block defeasible rules. Whatever the definition is, none of these systems characterized its outcomes.

\section{Acknowledgement}

This work benefited from the support of AMANDE ANR-13-BS02-0004 project of the French National Research Agency.

\section{References}

1. Amgoud, L., Besnard, P.: A Formal Characterization of the Outcomes of Rule-Based Argumentation Systems. In: 3rd International Conference on Scalable Uncertainty Management (SUM'13), pp. 78-91 (2013)

2. Amgoud, L., Caminada, M., Cayrol, C., Lagasquie, M.C., Prakken, H.: Towards a Consensual Formal Model: inference part. Deliverable of ASPIC project (2004)

3. Caminada, M., Amgoud, L.: On the evaluation of argumentation formalisms. Artificial Intelligence Journal, 171(5-6):286-310 (2007)

4. Caminada, M., Sá, S., Alcântara, J.: On the Equivalence between Logic Programming Semantics and Argumentation Semantics. In: 12th European Conference on Symbolic and Quantitative Approaches to Reasoning with Uncertainty, ECSQARU'13, pp. 97-108 (2013)

5. Cohen, A., García, A.J., Simari, G.R.: Backing and Undercutting in Defeasible Logic Programming. In: 11th European Conference on Symbolic and Quantitative Approaches to Reasoning with Uncertainty, ECSQARU'11, pp. 50-61 (2011)

6. Cohen, A., García, A.J., Simari, G.R.: Backing and Undercutting in Abstract Argumentation Frameworks. In: 7th International Conference on Foundations of Information and Knowledge Systems, FoIKS'12, pp. 107-123 (2012)

7. Dung, P.M.: On the acceptability of arguments and its fundamental role in nonmonotonic reasoning, logic programming and $n$-person games. Artificial Intelligence Journal, 77(2):321357 (1995)

8. García, A.J., Simari, G.R.: Defeasible logic programming: an argumentative approach. Theory and Practice of Logic Programming, 4(1-2):95-138 (2004)

9. Gebser, M. Gharib, M., Mercer, R., Schaub, T.: Monotonic Answer Set Programming. Journal of Logic and Computation, 19(4):539-564 (2009)

10. Gelfond, M., Lifschitz, V.: Classical negation in logic programs and disjunctive databases. New Generation Computing, 9:365-385 (1991)

11. Governatori, G., Maher, M.J., Antoniou, G., Billington, D.: Argumentation Semantics for Defeasible Logic. Journal of Logic and Computation, 14(5):675-702 (2004)

12. , Lukaszewicz, W.: Considerations on Default Logic : An Alternative Approach. Computational Intelligence, 4:1-16 (1988)

13. Pollock, J.L.: How to reason defeasibly. Artificial Intelligence Journal, 57(1):1-42 (1992)

14. Prakken, H.: An abstract framework for argumentation with structured arguments. Journal of Argument and Computation, 1(2):93-124 (2010)

15. Reiter, R.: A Logic for Default Reasoning. Artificial Intelligence Journal, 13(1-2): 81-132 (1980) 


\section{Appendix: Proofs}

Proof. of Proposition 1. Follows immediately from the fact that $\operatorname{Def}(d)=\emptyset$ for all $(d, x) \in \operatorname{Arg}((\mathcal{F}, \mathcal{S}, \emptyset))$.

Proof. of Theorem 1. Let $\mathcal{H}=\left(\operatorname{Arg}(\mathcal{T}), \mathcal{R}_{u}\right)$ be an argumentation system built over theory $\mathcal{T}=(\mathcal{F}, \mathcal{S}, \mathcal{D})$.

Coherence: Assume that $\operatorname{Ext}(\mathcal{H}) \neq \emptyset$ and let $\mathcal{E} \in \operatorname{Ext}(\mathcal{H})$. Assume that $\exists x \in$ $\operatorname{Concs}(\mathcal{E}) \cap \operatorname{Defs}(\operatorname{Th}(\mathcal{E}))$. Thus, $x \in \mathcal{L}^{\prime}$. Moreover, $\exists a, b \in \mathcal{E}$ such that $\operatorname{Conc}(a)=x$ and $x \in \operatorname{Defs}(\{b\})$. Then, $a \mathcal{R}_{u} b$. This contradicts the fact that $\mathcal{E}$ is conflict-free.

Closure under sub-arguments: Let $\mathcal{E} \in \operatorname{Ext}(\mathcal{H}), a=(d, x), b=\left(d^{\prime}, x^{\prime}\right) \in \operatorname{Arg}(\mathcal{T})$ such that $a \in \mathcal{E}, b \notin \mathcal{E}$ and $b \in \operatorname{Sub}(a)$.

Assume that $E \in \operatorname{Ext}_{s}(\mathcal{H})$. There exists $c \in \mathcal{E}$ such that $c \mathcal{R}_{u} b$. Let $c=\left(d^{\prime \prime}, x^{\prime \prime}\right)$. Then, $x^{\prime \prime} \in \operatorname{Def}\left(d^{\prime}\right)$ and thus $x^{\prime \prime} \in \operatorname{Def}(d)$ since $\operatorname{Def}\left(d^{\prime}\right) \subseteq \operatorname{Def}(d)$. Consequently, $c \mathcal{R}_{u} a$. This contradicts the fact that $\mathcal{E}$ is conflict-free.

Assume now that $\mathcal{E} \in \operatorname{Ext}_{n}(\mathcal{H})$, then $\mathcal{E} \cup\{b\}$ is conflicting. Then, there exists $c \in \mathcal{E}$ such that $c \mathcal{R}_{u} b$ or $b \mathcal{R}_{u} c$. Assume that $b \mathcal{R}_{u} c$. Then $x^{\prime} \in \mathcal{L}^{\prime}$. Since elements of $\mathcal{L}^{\prime}$ cannot be in the body of any rule then $a=b$, thus $a \mathcal{R}_{u} c$. This contradicts the fact that it is conflict-free. Assume now that $c \mathcal{R}_{u} b$. As above, it follows that $c \mathcal{R}_{u} a$ and this contradicts the fact that $\mathcal{E}$ is conflict-free.

Assume now that $\mathcal{E} \in \operatorname{Ext}_{p}(\mathcal{H})$. Since $b \notin \mathcal{E}$ then there are two cases: i) $\mathcal{E} \cup\{b\}$ is conflicting, i.e., there exists $c \in \mathcal{E}$ such that $c \mathcal{R}_{u} b$ or $b \mathcal{R}_{u} c$. As above, we get either $c \mathcal{R}_{u} a$ or $a \mathcal{R}_{u} c$. In both cases, $\mathcal{E}$ is not conflict-free and this contradicts the fact that it is a preferred extension. ii) $\mathcal{E}$ does not defend $b$. Thus, there exists $c=\left(d^{\prime \prime}, x^{\prime \prime}\right) \in \operatorname{Arg}(\mathcal{T})$ such that $c \mathcal{R}_{u} b$. Then, $x^{\prime \prime} \in \operatorname{Def}\left(d^{\prime}\right)$ and thus $x^{\prime \prime} \in \operatorname{Def}(d)$ meaning that $c \mathcal{R}_{u} a$. Since $\mathcal{E}$ is a preferred extension $\exists d \in \mathcal{E}$ such that $d \mathcal{R}_{u} c$. Thus, $\mathcal{E}$ defends $b$.

Proof. of Theorem 2. Let $\mathcal{H}=\left(\operatorname{Arg}(\mathcal{T}), \mathcal{R}_{u}\right)$ be an argumentation system built over theory $\mathcal{T}=(\mathcal{F}, \mathcal{S}, \mathcal{D})$.

Closure under strict rules: Let $\mathcal{E} \in \operatorname{Ext}_{p}(\mathcal{H})$. Assume that $x \in \operatorname{CN}((\operatorname{Concs}(\mathcal{E}), \mathcal{S}, \emptyset))$ and $x \notin \operatorname{Concs}(\mathcal{E})$. Let $X=\left\{x_{1}, \ldots, x_{n}\right\}$ be the minimal for set inclusion subset of $\operatorname{Concs}(\mathcal{E})$ such that $x \in \operatorname{CN}((X, \mathcal{S}, \emptyset))$. For each $x_{i}$, there exists $a_{i} \in \mathcal{E}$ such that Conc $\left(a_{i}\right)=x_{i}$. There exists a minimal derivation schema for $x$ using $a_{1}, \ldots, a_{n}$ and additional strict rules. Let $d$ be that derivation. $(d, x)$ is an argument and $(d, x) \notin \mathcal{E}$. There are two cases: i) $\mathcal{E} \cup\{(d, x)\}$ is conflicting, i.e, there exists $b=\left(d^{\prime}, x^{\prime}\right) \in \mathcal{E}$ such that $b \mathcal{R}_{u}(d, x)$ or $(d, x) \mathcal{R}_{u} b$. If $b \mathcal{R}_{u}(d, x)$, then $\operatorname{Conc}(b) \in \operatorname{Def}(d)$. However, $\operatorname{Def}(d)=\cup \operatorname{Def}\left(a_{i}\right)$. Thus, there exists $i \in\{1, \ldots, n\}$ such that $\operatorname{Conc}(b) \in \operatorname{Def}\left(a_{i}\right)$, i.e., $b \mathcal{R}_{u} a_{i}$. This contradicts the fact that $\mathcal{E}$ is conflict-free. If $(d, x) \mathcal{R}_{u} b$, the since $\mathcal{E}$ defends its elements, $\exists c \in \mathcal{E}$ such that $c \mathcal{R}_{u}(d, x)$, i.e., Conc $(c) \in \operatorname{Def}(d)$. Then, $\exists a_{i} \in$ $\operatorname{Sub}((d, x))$ such that $c \mathcal{R}_{u} a_{i}$. But, $a_{i} \in \mathcal{E}$. ii) $\mathcal{E}$ does not defend $(d, x)$. Let $b \in \operatorname{Arg}(\mathcal{T})$ such that $b \mathcal{R}_{u}(d, x)$. Then, $\operatorname{Conc}(b) \in \operatorname{Def}(d)$. Then, $b \mathcal{R} a_{i}$ for some $a_{i} \in \operatorname{Sub}((d, x))$ and $a_{i} \in \mathcal{E}$. Since $\mathcal{E}$ defends its elements, then $\mathcal{E}$ attacks $b$. The same reasoning holds for stable semantics since any stable extension is also preferred.

Consistency: Let $\mathcal{E} \in \operatorname{Ext}_{x}(\mathcal{H})$ with $x \in\{p, s\}$ and assume that $\operatorname{Concs}(\mathcal{E})$ is inconsistent. Thus, $\exists a, b \in \mathcal{E}$ such that $a=(d, x), b=\left(d^{\prime}, \neg x\right), d=\left\langle\left(x_{1}, r_{1}\right), \ldots,\left(x_{n}, r_{n}\right)\right\rangle$, $d^{\prime}=\left\langle\left(x_{1}^{\prime}\right), \ldots,\left(x_{m}^{\prime}, r_{m}\right)\right\rangle, x_{n}=x$ and $x_{m}^{\prime}=\neg x$. Moreover, $x, \neg x \in \mathcal{L}$. If $a$ 
and $b$ are both strict (i.e., $\operatorname{Def}(d)=\emptyset$ and $\operatorname{Def}\left(d^{\prime}\right)=\emptyset$ ), then $\operatorname{CN}((\mathcal{F}, \mathcal{S}, \emptyset))$ is inconsistent. Assume now that $\operatorname{CN}((\mathcal{F}, \mathcal{S}, \emptyset))$ is consistent. It follows that $a$ or/and $b$ is defeasible (i.e., $\operatorname{Def}(d) \neq \emptyset$ or/and $\operatorname{Def}(d) \neq \emptyset$ ). Assume that $a$ is defeasible. If $r_{n} \in \mathcal{D}$, then $\neg x \rightarrow r_{n} \in \mathcal{S}$ (since $\mathcal{T}$ is closed). Since $\mathcal{E}$ is closed under strict rules and $\neg x \in \operatorname{Concs}(\mathcal{E})$, then $r_{n} \in \operatorname{Concs}(\mathcal{E})$. Thus, $\operatorname{CN}(\operatorname{Th}(\mathcal{E})) \cap \operatorname{Defs}(\operatorname{Th}(\mathcal{E})) \neq$ $\emptyset$. This contradicts the fact that $\operatorname{Th}(\mathcal{E})$ is coherent. Assume now that $r_{n} \notin \mathcal{D}$. Let $r_{i} \in \operatorname{Def}(d)$ be such that for all $j>i, r_{j}$ is either a fact or a strict rule. By definition of a derivation, $r_{n} \in \mathcal{S}$. Let $r_{n}=y_{1}, \ldots, y_{l} \rightarrow x$. Since $\mathcal{S}$ is closed under contraposition, then for all $1 \leq j \leq l, y_{1}, \ldots, y_{j-1}, y_{j+1}, \ldots, y_{l} \rightarrow \neg y_{j} \in \mathcal{S}$. Moreover, there exists a minimal sub-derivation $d_{j}$ of $d$ for each $y_{j}$. Thus, $X_{j}=$ $\left\langle d_{1}, d_{j-1}, d_{j+1}, \ldots, d_{l}, d^{\prime},\left(\neg y_{j}, y_{1}, y_{j-1}, y_{j+1}, \ldots, y_{l} \rightarrow \neg y_{j}, \neg y_{j}\right)\right\rangle$ is a derivation of $\neg y_{j}$. Since arguments are minimal, then $\left(X_{j}, \neg y_{j}\right) \in \operatorname{Arg}(\mathcal{T})$. Note that $\left(d_{i}, y_{i}\right) \in$ $\operatorname{Sub}(a)$. Since $\mathcal{H}$ is closed under sub-arguments, then $\left(d_{i}, y_{i}\right) \in \mathcal{E}$ and thus $y_{i} \in$ $\operatorname{Concs}(\mathcal{E})$. Since $\mathcal{H}$ is closed under strict rules, $\neg y_{j} \in \operatorname{Concs}(\mathcal{E})$ for all $j=1, \ldots, l$.

The same reasoning holds for each strict rule $y_{1}, \ldots, y_{l} \rightarrow y$ between $r_{i}$ and $r_{n}$. Indeed, $\neg y_{i} \in \operatorname{Concs}(\mathcal{E})$ for all $i=1, \ldots, l$. By definition of derivation, there exists a strict rule $r$ after $r_{i}$ such that $\operatorname{Head}\left(r_{i}\right) \in \operatorname{Body}(r)$ thus $\neg \operatorname{Head}\left(r_{i}\right) \in \operatorname{Concs}(\mathcal{E})$. Thus, $\neg$ Head $\left(r_{i}\right) \rightarrow r_{i} \in \mathcal{S}$. Since $\mathcal{H}$ is closed under strict rules, $r_{i} \in \operatorname{Concs}(\mathcal{E})$. But, $r_{i} \in \operatorname{Def} \mathbf{s}(\mathcal{E})$ (since $r_{i} \in \operatorname{Def}(d)$ ). This contradicts the fact that $\operatorname{Th}(\mathcal{E})$ is coherent.

Proof. of Corollary 1. Let $\mathcal{H}=\left(\operatorname{Arg}(\mathcal{T}), \mathcal{R}_{u}\right)$ be an argumentation system built over a closed theory $\mathcal{T}=(\mathcal{F}, \mathcal{S}, \mathcal{D})$ s.t. $\operatorname{Ext}(\mathcal{H}) \neq \emptyset$. Assume that Output $(\mathcal{H})$ is inconsistent then $\exists x, \neg x \in \operatorname{Output}(\mathcal{H})$. Thus, for all $\mathcal{E} \in \operatorname{Ext}(\mathcal{H}), x, \neg x \in \operatorname{Concs}(\mathcal{E})$. From Theorem 1, this is only possible if $\mathrm{CN}((\mathcal{F}, \mathcal{S}, \emptyset))$ is inconsistent.

Since $\mathrm{CN}$ is monotonic, Output $(\mathcal{H}) \subseteq \mathrm{CN}(\operatorname{Output}(\mathcal{H}))$. Let $x \in \mathrm{CN}(\operatorname{Output}(\mathcal{H}))$ and assume that $x \notin \operatorname{Output}(\mathcal{H})$, thus, there exists $\mathcal{E} \in \operatorname{Ext}(\mathcal{H})$ such that $x \notin$ $\operatorname{Concs}(\mathcal{E})$. This contradicts Theorem 1 .

Proof. of Theorem 3. Let $\mathcal{H}=\left(\operatorname{Arg}(\mathcal{T}), \mathcal{R}_{u}\right)$ be a system built over a theory $\mathcal{T}$.

- Let $\mathcal{E} \in \operatorname{Ext}_{n}(\mathcal{H})$ and let $\mathcal{O}=\operatorname{Th}(\mathcal{E})$. It is clear that $\mathcal{O}$ is uniquely determined from $\mathcal{E}$. Let us show that $\mathcal{O}$ is an option. $\mathcal{O}=\left(\mathcal{F}^{\prime}, \mathcal{S}^{\prime}, \mathcal{D}^{\prime}\right)$ such that $\mathcal{F}^{\prime}=\bigcup_{(d, x) \in \mathcal{E}} \operatorname{Facts}(d), F^{\prime}=\bigcup_{(d, x) \in \mathcal{E}} \operatorname{Strict}(d)$ and $\mathcal{D}^{\prime}=\bigcup_{(d, x) \in \mathcal{E}} \operatorname{Def}(d)$.

- It is obvious that $\mathcal{S}^{\prime} \subseteq \mathcal{S}$ and $\mathcal{D}^{\prime} \subseteq \mathcal{D}$. Now, for every $x \in \mathcal{F}$ there is an argument $(\langle(x, \emptyset)\rangle, x) \in \operatorname{Arg}(\mathcal{T})$. By definiteion of undercutting, such argument has no conflict with any other argument. Thus, all arguments of this form belong to every naive extension, i.e., $\mathcal{F}^{\prime}=\mathcal{F}$.

- For the sake of contradiction, suppose that $\exists x \in \operatorname{CN}\left(\mathcal{F}^{\prime}, \mathcal{S}^{\prime}, \mathcal{D}^{\prime}\right)$ s.t. $x \in \mathcal{D}^{\prime}$. Let $d$ be a minimal derivation of $x$ in $\mathcal{O}$. Thus $(d, x)$ is an argument of $\mathcal{E}$. since $x \in$ $\mathcal{D}^{\prime}$ then, from the definition of $\operatorname{Th}(\mathcal{E}), x$ must be used in at least an argument of $\mathcal{E}$, say $\left(d^{\prime}, x^{\prime}\right)$, i.e., $x \in d^{\prime}$. Therefore, $(d, x) \mathcal{R}_{u}\left(d^{\prime}, x^{\prime}\right)$. Contradiction with conflict-freeness of $\mathcal{E}$.

- Let $r \in \mathcal{S}^{\prime} \cup \mathcal{D}^{\prime}$. $r$ is used in at least one argument, say $a$, of $\mathcal{E}$. So, $a$ has a subargument $b=\left(\left\langle\left(x_{1}, r_{1}\right), \ldots\left(x_{n}, r_{n}\right)\right\rangle, x_{n}\right)$ with $r_{n}=r$ and $x_{n}=\operatorname{Head}(r)$. By closeness ander sub-arguments, $b$ is also an argument of $\mathcal{E}$. From the definition of derivation schema, for every $x \in \operatorname{Body}(r), x=x_{i}$ for some $i$ s.t. $1 \leq i<n$. 
Thus, there is a sub-argument of $b$, and hence an $\operatorname{argument}$ in $\mathcal{E}$ and a derivation in $\mathcal{O}$, for every $x \in \operatorname{Body}(r)$. This means that for every $x \in \operatorname{Body}(r), x \in$ $\mathrm{CN}(\mathcal{O})$, i.e., $\operatorname{bod} y(r) \subseteq \mathrm{CN}(\mathcal{O})$.

- Suppose that $\exists \mathcal{S}^{\prime \prime}, \mathcal{D}^{\prime \prime}$ s.t. $\left(\mathcal{F}^{\prime}, \mathcal{S}^{\prime}, \mathcal{D}^{\prime}\right) \sqsubset\left(\mathcal{F}^{\prime}, \mathcal{S}^{\prime \prime}, \mathcal{D}^{\prime \prime}\right)$ and $\left(\mathcal{F}^{\prime}, \mathcal{S}^{\prime \prime}, \mathcal{D}^{\prime \prime}\right)$ satisfies the previous conditions. Every rule $r \in\left(\mathcal{S}^{\prime \prime} \cup \mathcal{D}^{\prime \prime}\right) \backslash\left(\mathcal{S}^{\prime} \cup \mathcal{D}^{\prime}\right)$, there is at least an argument $a=(d, x)$ s.t. $r \in \operatorname{Strict}(d) \cup \operatorname{Def}(d)$. Clearly, $a \notin \mathcal{E}$. But from the coherence of $\left(\mathcal{F}^{\prime}, \mathcal{S}^{\prime \prime}, \mathcal{D}^{\prime \prime}\right)$ it must be the case that $\nexists b \in \mathcal{E}$ s.t. $a \mathcal{R}_{u} b$ or $b \mathcal{R}_{u} a$. Indeed, suppose for example that $a \mathcal{R}_{u} b$ and that $b=\left(d^{\prime}, x^{\prime}\right)$, then $x \in d^{\prime}$. That is, $x \in \operatorname{CN}\left(\mathcal{F}^{\prime}, \mathcal{S}^{\prime \prime}, \mathcal{D}^{\prime \prime}\right)$ and $x \in \mathcal{D}^{\prime \prime}$ which contradicts the coherence of $\left(\mathcal{F}^{\prime}, \mathcal{S}^{\prime \prime}, \mathcal{D}^{\prime \prime}\right)$. We can show in a similar way that it must not be the case that $b \mathcal{R}_{u} a$. But, it this case $\mathcal{E} \cup a$ is conflict-free. Contradiction with the fact that $\mathcal{E}$ is a naive extension.

- Let $\mathcal{E}, \mathcal{E}^{\prime} \in \operatorname{Ext}_{n}(\mathcal{H})$ and Option $(\mathcal{E})=\operatorname{Option}\left(\mathcal{E}^{\prime}\right)$. Let us show that $\mathcal{E} \subseteq \mathcal{E}^{\prime}$. Let $a=(d, x) \in \mathcal{E}$. Then, $d$ is a derivation for $x$ in $\operatorname{Option}(\mathcal{E})$. Suppose that $a \notin \mathcal{E}^{\prime}$. Then $d$ is not a derivation for $x$ in $\operatorname{Option}\left(\mathcal{E}^{\prime}\right)$. Contradiction, since Option $(\mathcal{E})=$ Option $\left(\mathcal{E}^{\prime}\right)$. We show similarly that $\mathcal{E}^{\prime} \subseteq \mathcal{E}$.

- Let $\mathcal{E} \in \operatorname{Ext}_{n}(\mathcal{H})$. Since $\operatorname{Option}(\mathcal{E})=\operatorname{Th}(\mathcal{E})$ and from the definition of functions Th and $\operatorname{Arg}$ it is obvious that $\mathcal{E} \subseteq \operatorname{Arg}(\operatorname{Option}(\mathcal{E}))$. Now let $a=(d, x) \in$ $\operatorname{Arg}(\operatorname{Option}(\mathcal{E}))$. This means that $a=(d, x)$ is constructed from Option $(\mathcal{E})$. So, $x \in \operatorname{CN}(\operatorname{Option}(\mathcal{E}))$ and $\operatorname{Def}(d) \subseteq \operatorname{Defs}(\operatorname{Option}(\mathcal{E}))$. Suppose that $a \notin \mathcal{E}$. Since $\mathcal{E}$ is a naive extension then there is $b=\left(d^{\prime}, x^{\prime}\right) \in \mathcal{E}$ such that $a \mathcal{R}_{u} b$ or $b \mathcal{R}_{u} a$. From $b \in \mathcal{E}$ we easily deduce that $x^{\prime} \in \operatorname{CN}($ Option $(\mathcal{E}))$ and $\operatorname{Def}\left(d^{\prime}\right) \subseteq$ $\operatorname{Defs}(\operatorname{Option}(\mathcal{E}))$. But then, from $a \mathcal{R}_{u} b$ or $b \mathcal{R}_{u} a$, Option $(\mathcal{E})$ must be incoherent. Contradiction with the fact that $\operatorname{Option}(\mathcal{E})$ is an option.

Proof. of Theorem 4. Let $\mathcal{H}=\left(\operatorname{Arg}(\mathcal{T}), \mathcal{R}_{u}\right)$ be a system built over a theory $\mathcal{T}$.

- Let $\mathcal{O}=\left(\mathcal{F}, \mathcal{S}^{\prime}, \mathcal{D}^{\prime}\right) \in \operatorname{Opt}(\mathcal{T})$ and let $\mathcal{E}=\operatorname{Arg}(\mathcal{O})$. We prove that $\mathcal{E}$ is a maximal conflict-free set of $\operatorname{Arg}(\mathcal{T})$.

Suppose that there is two arguments $a=(d, x)$ and $b=\left(d^{\prime}, x^{\prime}\right)$ in $\mathcal{E}$ s.t. $a \mathcal{R}_{u} b$, i.e., $x \in \operatorname{Def}\left(d^{\prime}\right)$. But since $d$ and $d^{\prime}$ are derivation schemas for $x$ and $x^{\prime}$ respectively in $\mathcal{O}$ we have: $x \in \operatorname{CN}(\mathcal{O})$ and $\operatorname{Def}\left(d^{\prime}\right) \subseteq \mathcal{D}^{\prime}$, so $x \in \mathcal{D}^{\prime}$. Contradiction with the coherence of option $\mathcal{O} \cdot \mathcal{E}=\operatorname{Arg}(\mathcal{O})$ is conflict-free.

Now, suppose that $\mathcal{E}$ is not maximal. Thus there is $\mathcal{E}^{\prime} \subseteq \operatorname{Arg}(\mathcal{T})$ s.t. $\mathcal{E} \subset \mathcal{E}^{\prime}$ and $\mathcal{E}^{\prime}$ is a naive extension of $\mathcal{H}$. From theorem $3 \operatorname{Option}\left(\mathcal{E}^{\prime}\right)=\operatorname{Th}\left(\mathcal{E}^{\prime}\right)=\mathcal{O}^{\prime}$ is an option of $\mathcal{T}$. Let $\mathcal{O}^{\prime}=\left(\mathcal{F}, \mathcal{S}^{\prime \prime}, \mathcal{D}^{\prime \prime}\right)$. Since all the arguments that uses exclusively rules from $\mathcal{S}^{\prime} \cup \mathcal{D}^{\prime}$ belong to $\mathcal{E}$, every argument $\mathcal{E}^{\prime} \backslash \mathcal{E}$ uses at least a rule $r$ which is not in $\mathcal{S}^{\prime} \cup \mathcal{D}^{\prime}$. So, either $\left(\mathcal{S}^{\prime} \subset \mathcal{S}^{\prime \prime}\right)$ or $\left(\mathcal{D}^{\prime} \subset \mathcal{D}^{\prime \prime}\right)$ or both, i.e, $\mathcal{O} \sqsubset \mathcal{O}^{\prime}$. contradiction with the fact that $\mathcal{O}$ is an option of $\mathcal{T}$.

- Let $\mathcal{O}=\left(\mathcal{F}, \mathcal{S}^{\prime}, \mathcal{D}^{\prime}\right) \in \operatorname{Opt}(\mathcal{T})$ and let Option $(\operatorname{Arg}(\mathcal{O}))=\left(\mathcal{F}^{\prime \prime}, \mathcal{S}^{\prime \prime}, \mathcal{D}^{\prime \prime}\right) . \mathcal{F}^{\prime \prime}=$ $\mathcal{F}$ follows from the fact that $\operatorname{Arg}(\mathcal{O})$ contains every argument $(\langle x, \emptyset\rangle, x)$. Let $x \in$ $\mathcal{S}^{\prime \prime}$ (resp. $x \in \mathcal{D}^{\prime \prime}$ ). $x$ is used in at least an argument of $\operatorname{Arg}(\mathcal{O})$, so $x \in \mathcal{S}^{\prime}$ (resp. $x \in \mathcal{D}^{\prime}$ ). Thus we have: $\mathcal{S}^{\prime \prime} \subseteq \mathcal{S}^{\prime}$ and $\mathcal{D}^{\prime \prime} \subseteq \mathcal{D}^{\prime}$. Inversely, let $x \in \mathcal{S}^{\prime}$ (resp. $x \in \mathcal{D}^{\prime}$ ), since $\operatorname{Body}(x) \subseteq \operatorname{CN}(\mathcal{O})$ (from the definition of an option), $x$ must be used in at least one argument of $\operatorname{Arg}(\mathcal{O})$. Thus $x \in \mathcal{F}^{\prime \prime}$ (resp. $x \in \mathcal{D}^{\prime \prime}$ ). So, $\mathcal{F}^{\prime} \subseteq$ 
$\mathcal{F}^{\prime \prime}$ and $\mathcal{D}^{\prime} \subseteq \mathcal{D}^{\prime \prime}$. In summary, $\mathcal{F}=\mathcal{F}^{\prime \prime}, \mathcal{S}^{\prime}=\mathcal{S}^{\prime \prime}$ and $\mathcal{D}^{\prime}=\mathcal{D}^{\prime \prime}$, i.e., $\mathcal{O}=$ $\operatorname{Option}(\operatorname{Arg}(\mathcal{O}))$.

- Let $\mathcal{O}_{1}=\left(\mathcal{F}, \mathcal{S}_{1}^{\prime}, \mathcal{D}_{1}^{\prime}\right)$ and $\mathcal{O}_{2}=\left(\mathcal{F}, \mathcal{S}_{2}^{\prime}, \mathcal{D}_{2}^{\prime}\right)$ be two options. Suppose that $\mathcal{O}_{1} \neq$ $\mathcal{O}_{2}$, i.e., either $S_{1}^{\prime} \neq S_{2}^{\prime}$ or $D_{1}^{\prime} \neq D_{2}^{\prime}$ or both. Suppose that $S_{1}^{\prime} \neq S_{2}^{\prime}$. It means that either there is $x$ s.t. $x \in \mathcal{S}_{1}^{\prime}$ and $x \notin \mathcal{S}_{2}^{\prime}$ or there is $x$ s.t. $x \in \mathcal{S}_{2}^{\prime}$ and $x \notin \mathcal{S}_{1}^{\prime}$. Suppose the first case. Then, $x$ is used in at least an $\operatorname{argument}$ of $\operatorname{Arg}\left(\mathcal{O}_{1}\right)$ and never used in $\operatorname{Arg}\left(\mathcal{O}_{2}\right)$. So, $\operatorname{Arg}\left(\mathcal{O}_{1}\right) \neq \operatorname{Arg}\left(\mathcal{O}_{2}\right)$. By a similar reasoning, we obtain the same conclusion for the other case (there is $x$ s.t. $x \in \mathcal{S}_{2}^{\prime}$ and $x \notin \mathcal{S}_{1}^{\prime}$ ) and for the case of defeasible rules.

Proof. of Corollary 2. Follows immediately from the bijection between options and naive extensions(theorems 3 - 4).

Proof. of Corollary 3. Follows immediately from the bijection between options and naive extensions(theorems 3 - 4).

Proof. of Theorem 5. Let $\mathcal{H}=\left(\operatorname{Arg}(\mathcal{T}), \mathcal{R}_{u}\right)$ s.t. $\operatorname{Ext}_{s}(\mathcal{H}) \neq \emptyset$.

- Let us show that for all $\mathcal{E} \in \operatorname{Ext}_{s}(\mathcal{H})$, there is a unique $\mathcal{O} \in \operatorname{SOpt}(\mathcal{T})$ s.t. $\operatorname{Th}(\mathcal{E}) \sqsubseteq$ $\mathcal{O}$ and $\operatorname{Concs}(\mathcal{E})=\operatorname{CN}(\mathcal{O})$.

Let $\mathcal{E} \in \operatorname{Ext}_{s}(\mathcal{H})$ and let $\operatorname{Th}(\mathcal{E})=\left(\mathcal{F}^{\prime}, \mathcal{S}^{\prime}, \mathcal{D}^{\prime}\right)$. We can show that $\mathcal{F}^{\prime}=\mathcal{F}$ in a similar way as in Theorem 3 , first point. We take $\mathcal{O}=\left(\mathcal{F}, \mathcal{S}, \mathcal{D}^{\prime}\right)$ (we complete $\mathcal{S}^{\prime}$ by the remaining strict rules). Clearly, $\mathcal{O}$ is uniquely determined from $\mathcal{E}$. We have that $\operatorname{Concs}(\mathcal{E})=\operatorname{CN}(\operatorname{Th}(\mathcal{E}))$. Let us show that: $\operatorname{CN}\left(\left(\mathcal{F}, \mathcal{S}, \mathcal{D}^{\prime}\right)\right)=\operatorname{CN}(\operatorname{Th}(\mathcal{E}))$. To do so, it suffices to show that every rule of $r \in \mathcal{S} \backslash \mathcal{S}^{\prime}$ is not applicable in $\left(\mathcal{F}, \mathcal{S}^{\prime}, \mathcal{D}^{\prime}\right)$. Suppose for the sake of contradiction that there is $r \in \mathcal{S} \backslash \mathcal{S}^{\prime}$ s.t. $r$ is applicable in $\left(\mathcal{F}, \mathcal{S}^{\prime}, \mathcal{D}^{\prime}\right)$. Thus, there is a minimal derivation in $\left(\mathcal{F}, \mathcal{S}^{\prime}, \mathcal{D}^{\prime}\right)$ for $\operatorname{Head}(r))$ using $r$ as a last rule: $\langle d,(x, r)\rangle$ s.t. $x=\operatorname{Head}(r), \operatorname{Def}(d) \subseteq \mathcal{D}^{\prime}$ and Strict $(d) \subseteq \mathcal{S}^{\prime}$. Thus, $a=(\langle d,(x, r)\rangle, x)$ is an argument outside $\mathcal{E}$ but since $\mathcal{E}$ is a stable extension, there is $b \in \mathcal{E}$ s.t. $b \mathcal{R}_{u} a$. So, there is a sub-argument of a: $a^{\prime}=\left(\left\langle d^{\prime},\left(x^{\prime}, r^{\prime}\right)\right\rangle, x^{\prime}\right)$ with $x^{\prime} \in \mathcal{D}^{\prime}$ and $b=\left(d^{\prime \prime}, x^{\prime}\right)$. However since $a^{\prime} \in \mathcal{E}$ (because it uses only rules from $\left.S^{\prime} \cup D^{\prime}\right)$, this means that $\mathcal{E}$ is not conflict-free. Contradiction. Now let us prove that $\mathcal{O}=\left(\mathcal{F}, \mathcal{S}, \mathcal{D}^{\prime}\right)$ is a strong option.

- It is obvious that $\mathcal{D}^{\prime} \subseteq \mathcal{D}$

- Similar to the proof of point 2 in Theroem 3.

- Similar to the proof of point 3 in Theroem 3.

- Suppose that $\exists r \notin \mathcal{D}^{\prime}$ s.t. $r \notin \operatorname{CN}\left(\mathcal{F}, \mathcal{S}, \mathcal{D}^{\prime}\right)$ and $\forall x \in \operatorname{Body}(r), x \in \operatorname{CN}\left(\mathcal{F}, \mathcal{S}, \mathcal{D}^{\prime}\right)$. Let $\operatorname{Body}(r)=\left\{x_{1}, \ldots, x_{k}\right\}$ and $\operatorname{Head}(r)=y$. Since for $1 \leq i \leq k$, $x_{i} \in \mathrm{CN}\left(\mathcal{F}, \mathcal{S}, \mathcal{D}^{\prime}\right)$, then there is an argument $a_{i}=\left(d_{i}, x i\right) \in \mathcal{E}(1 \leq i \leq k)$ for each $x_{i}$. Thus, we can construct an argument $a$ for $y$ using $r$ as last rule, i.e., $a=(\langle d,(y, r)\rangle, y)$ where $\operatorname{Facts}(d)=\bigcup_{i} \operatorname{Facts}\left(d_{i}\right)$, $\operatorname{Strict}(d)=$ $\bigcup_{i} \operatorname{Strict}\left(d_{i}\right)$ and $\operatorname{Def}(d)=\bigcup_{i} \operatorname{Def}\left(d_{i}\right)$. Since $r \notin \mathcal{D}^{\prime}, a \notin \mathcal{E}$, so there is $b=\left(d^{\prime}, x^{\prime}\right) \in \mathcal{E}$ s.t. $b \mathcal{R}_{u} a$, i.e., $x^{\prime} \in \operatorname{Def}(d) \cup\{r\}$. Sincd $r \notin \operatorname{CN}\left(\mathcal{F}, \mathcal{S}, \mathcal{D}^{\prime}\right)$, it cannot be the case that $x^{\prime}=r$, thus $x^{\prime} \in \operatorname{Def}(d)$, so $x^{\prime} \in \operatorname{Def}\left(d_{i}\right)$ for some $i$ s.t. $1 \leq i \leq k$. This means that $b \mathcal{R}_{u} a_{i}$ which contradicts the conflict-freeness of $\mathcal{E}$. 
- Let $\mathcal{E}, \mathcal{E}^{\prime} \in \operatorname{Ext}_{s}(\mathcal{H})$ and $\operatorname{SOption}(\mathcal{E})=\operatorname{SOption}\left(\mathcal{E}^{\prime}\right)$. Let us show that $\mathcal{E} \subseteq \mathcal{E}^{\prime}$. Let $a=(d, x) \in \mathcal{E}$. Then, $d$ is a derivation for $x$ in $\operatorname{SOption}(\mathcal{E})$. Suppose that $a \notin \mathcal{E}^{\prime}$. Then $d$ is not a derivation for $x$ in $\operatorname{SOption}\left(\mathcal{E}^{\prime}\right)$. Contradiction, since $\operatorname{SOption}(\mathcal{E})=\operatorname{SOption}\left(\mathcal{E}^{\prime}\right)$. We show similarly that $\mathcal{E}^{\prime} \subseteq \mathcal{E}$.

- Let $\mathcal{E} \in \operatorname{Ext}_{s}(\mathcal{H})$. Since $\operatorname{Th}(\mathcal{E}) \sqsubseteq \operatorname{SOption}(\mathcal{E})$ and from the definition of functions Th and $\operatorname{Arg}$ it is obvious that $\mathcal{E} \subseteq \operatorname{Arg}(\operatorname{SOption}(\mathcal{E}))$. Now let $a=(d, x) \in$ $\operatorname{Arg}(\operatorname{SOption}(\mathcal{E})) . a=(d, x)$ is constructed from $\operatorname{SOption}(\mathcal{E})$. So, $\operatorname{Def}(d) \subseteq$ Defs(SOption $(\mathcal{E}))$. Suppose that $a \notin \mathcal{E}$. Since $\mathcal{E}$ is a stable extension then there is $b=\left(d^{\prime}, x^{\prime}\right) \in \mathcal{E}$ such that $b \mathcal{R}_{u} a$. From $b \in \mathcal{E}$ we easily deduce that $x^{\prime} \in$ $\mathrm{CN}(\operatorname{SOption}(\mathcal{E}))$. But then, from $b \mathcal{R}_{u} a$, SOption $(\mathcal{E})$ must be incoherent. Contradiction with the fact that $\operatorname{SOption}(\mathcal{E})$ is a strong option.

Proof. of Theorem 6. Let $\mathcal{H}=\left(\operatorname{Arg}(\mathcal{T}), \mathcal{R}_{u}\right)$ s.t. $\operatorname{Ext}_{s}(\mathcal{H}) \neq \emptyset$.

- Let $\mathcal{O}=\left(\mathcal{F}, \mathcal{S}, \mathcal{D}^{\prime}\right) \in \operatorname{SOpt}(\mathcal{T})$ and let $\mathcal{E}=\operatorname{Arg}(\mathcal{O})$. We prove that $\mathcal{E}$ is conflictfree and $\forall b \in \operatorname{Arg}(\mathcal{T}) \backslash \mathcal{E}, \exists a \in \mathcal{E}$ s.t. $a \mathcal{R}_{u} b$.

Suppose that there is two argument $a=(d, x)$ and $b=\left(d^{\prime}, x^{\prime}\right)$ in $\mathcal{E}$ s.t. $a \mathcal{R}_{u} b$, i.e., $x \in \operatorname{Def}\left(d^{\prime}\right)$. But since $d$ and $d^{\prime}$ are derivation schemas for $x$ and $x^{\prime}$ respectively in $\mathcal{O}$ we have: $x \in \operatorname{CN}(\mathcal{O})$ and $\operatorname{Def}\left(d^{\prime}\right) \subseteq \mathcal{D}^{\prime}$, so $x \in \mathcal{D}^{\prime}$. Contradiction with the coherence of strong option $\mathcal{O}$. So, $\mathcal{E}$ is conflict-free.

Now, let us show that: $\forall b \in \operatorname{Arg}(\mathcal{T}) \backslash \mathcal{E}, \exists a \in \mathcal{E}$ s.t. $a \mathcal{R}_{u} b$. Let $b=(d, x) \notin \mathcal{E}$. Clearly, $d$ uses at least a defeasible rule $r(r \in \operatorname{Def}(d))$ s.t. $r \notin \mathcal{D}^{\prime}$. From the definition of a strong option, we have two possible cases. The first case is that $r \in \operatorname{CN}\left(\mathcal{F}, \mathcal{S}, \mathcal{D}^{\prime}\right)$, so there is a minimal derivation $d^{\prime}$ for $r$ in $\mathcal{O}$, i.e., $a=\left(d^{\prime}, r\right)$ is an argument of $\mathcal{E}$. Clearly $a \mathcal{R}_{u} b$ since $r \in \operatorname{Def}(d)$. The second case is that $\exists x_{1} \in \operatorname{Body}(r)$ s.t. $x_{1} \notin \mathrm{CN}(\mathcal{O})$. Let $b_{1}=\left(d_{1}, x_{1}\right)$ be a sub-argument of $b$. Since $x_{1} \notin \mathrm{CN}(\mathcal{O})$, there is no derivation of $x_{1}$ in $\mathcal{O}$, so $b_{1} \notin \mathcal{E}$. Thus, $d_{1}$ uses at least a defeasible rule $r_{1}\left(r_{1} \in \operatorname{Def}\left(d_{1}\right)\right)$ s.t. $r_{1} \notin \mathcal{D}^{\prime}$. Again, from the definition of a strong option, we have two possible cases. The first case is that $r_{1} \in \operatorname{CN}\left(\mathcal{F}, \mathcal{S}, \mathcal{D}^{\prime}\right)$, so there is a minimal derivation $d^{\prime \prime}$ for $r_{1}$ in $\mathcal{O}$, i.e., $a=\left(d^{\prime \prime}, r_{1}\right)$ is an argument of $\mathcal{E}$. Clearly $a \mathcal{R}_{u} b_{1}$ since $r \in \operatorname{Def}\left(d_{1}\right)$, hence, so $a \mathcal{R}_{u} b$. The second case is that $\exists x_{2} \in \operatorname{Body}\left(r_{1}\right)$ s.t. $x_{2} \notin \mathrm{CN}(\mathcal{O})$. Let $b_{2}=\left(d_{2}, x_{2}\right)$ be a sub-argument of $b_{1}$. Since $x_{2} \notin \mathrm{CN}(\mathcal{O})$, there is no derivation of $x_{2}$ in $\mathcal{O}$, so $b_{2} \notin \mathcal{E}$. Thus, $d_{2}$ uses at least a defeasible rule $r_{2}\left(r_{2} \in \operatorname{Def}\left(d_{2}\right)\right)$ s.t. $r_{2} \notin \mathcal{D}^{\prime}$, an so one. Since the set of arguments $\operatorname{Arg}(\mathcal{T}) \backslash \mathcal{E}$ is finite, it must exist a sub-argument $b_{k}$ of $b$ such that $a \mathcal{R}_{u} b_{k}$ and hence $a \mathcal{R}_{U} b$ for some $a \in \mathcal{E}$.

- Let $\mathcal{O}=\left(\mathcal{F}, \mathcal{S}, \mathcal{D}^{\prime}\right) \in \operatorname{SOpt}(\mathcal{T})$ and SOption $(\operatorname{Arg}(\mathcal{O}))=\left(\mathcal{F}^{\prime \prime}, \mathcal{S}^{\prime \prime}, \mathcal{D}^{\prime \prime}\right) . \mathcal{F}^{\prime \prime}=$ $\mathcal{F}$ follows from the fact that $\operatorname{Arg}(\mathcal{O})$ contains every argument $(\langle x, \emptyset\rangle, x) . \mathcal{S}^{\prime \prime}=\mathcal{S}$ follows from the definition of SOption. Let us show that $\mathcal{D}^{\prime \prime}=\mathcal{D}^{\prime}$. Let $x \in \mathcal{D}^{\prime \prime}$. $x$ is used in at least an argument of $\operatorname{Arg}(\mathcal{O})$, so $x \in \mathcal{D}^{\prime}$. Thus we have: $\mathcal{D}^{\prime \prime} \subseteq \mathcal{D}^{\prime}$. Inversely, let $x \in \mathcal{D}^{\prime}$, since $\operatorname{Body}(x) \subseteq \operatorname{CN}(\mathcal{O})$ (from the definition of a strong option), $x$ must be used in at least one argument of $\operatorname{Arg}(\mathcal{O})$. Thus $x \in \mathcal{D}^{\prime \prime}$. So, $\mathcal{D}^{\prime} \subseteq$ $\mathcal{D}^{\prime \prime}$. In summary, $\mathcal{F}=\mathcal{F}^{\prime \prime}, \mathcal{S}=\mathcal{S}^{\prime \prime}$ and $\mathcal{D}^{\prime}=\mathcal{D}^{\prime \prime}$, i.e., $\mathcal{O}=\operatorname{SOption}(\operatorname{Arg}(\mathcal{O}))$.

- Let $\mathcal{O}_{1}=\left(\mathcal{F}, \mathcal{S}_{1}, \mathcal{D}_{1}^{\prime}\right)$ and $\mathcal{O}_{2}=\left(\mathcal{F}, \mathcal{S}_{2}, \mathcal{D}_{2}^{\prime}\right)$ be two strong options. Suppose that $\mathcal{O}_{1} \neq \mathcal{O}_{2}$, i.e., $D_{1}^{\prime} \neq D_{2}^{\prime}$. It means that either there is $x$ s.t. $x \in \mathcal{D}_{1}^{\prime}$ and $x \notin \mathcal{D}_{2}^{\prime}$ or there is $x$ s.t. $x \in \mathcal{D}_{2}^{\prime}$ and $x \notin \mathcal{D}_{1}^{\prime}$. Suppose the first case. Then, $x$ is used in at least 
an argument of $\operatorname{Arg}\left(\mathcal{O}_{1}\right)$ and never used in $\operatorname{Arg}\left(\mathcal{O}_{2}\right)$. So, $\operatorname{Arg}\left(\mathcal{O}_{1}\right) \neq \operatorname{Arg}\left(\mathcal{O}_{2}\right)$. By a similar reasoning, we obtain the same conclusion for the other case (there is $x$ s.t. $x \in \mathcal{D}_{2}^{\prime}$ and $\left.x \notin \mathcal{D}_{1}^{\prime}\right)$.

Proof. of Corollary 4. Follows immediately from the bijection between strong options and stable extensions(theorems 5 - 6).

Proof. of Corollary 5. Follows immediately from the bijection between strong options and stable extensions(theorems 5 - 6).

Proof. of Theorem 7. Let $\mathcal{H}=\left(\operatorname{Arg}(\mathcal{T}), \mathcal{R}_{u}\right)$ s.t. $\operatorname{Ext}_{p}(\mathcal{H}) \neq \emptyset$.

- Let us show that for all $\mathcal{E} \in \operatorname{Ext}_{p}(\mathcal{H})$, there is a unique $\mathcal{O} \in \operatorname{POpt}(\mathcal{T})$ s.t. $\operatorname{Th}(\mathcal{E}) \sqsubseteq$ $\mathcal{O}$ and $\operatorname{Concs}(\mathcal{E})=\operatorname{CN}(\mathcal{O})$.

Let $\mathcal{E} \in \operatorname{Ext}_{p}(\mathcal{H})$ and let $\operatorname{Th}(\mathcal{E})=\left(\mathcal{F}^{\prime}, \mathcal{S}^{\prime}, \mathcal{D}^{\prime}\right)$. We can show that $\mathcal{F}^{\prime}=\mathcal{F}$ in a similar way as in Theorem 3 , first point. We take $\mathcal{O}=\left(\mathcal{F}, \mathcal{S}, \mathcal{D}^{\prime}\right)$ (we complete $\mathcal{S}^{\prime}$ by the remaining strict rules). Clearly, $\mathcal{O}$ is uniquely determined from $\mathcal{E}$. We have that $\operatorname{Concs}(\mathcal{E})=\operatorname{CN}(\operatorname{Th}(\mathcal{E}))$. Let us show that: $\operatorname{CN}\left(\left(\mathcal{F}, \mathcal{S}, \mathcal{D}^{\prime}\right)\right)=\operatorname{CN}(\operatorname{Th}(\mathcal{E}))$. To do so, it suffices to show that every rule of $r \in \mathcal{S} \backslash \mathcal{S}^{\prime}$ is not applicable in $\left(\mathcal{F}, \mathcal{S}^{\prime}, \mathcal{D}^{\prime}\right)$. Suppose for the sake of contradiction that there is $r \in \mathcal{S} \backslash \mathcal{S}^{\prime}$ s.t. $r$ is applicable in $\left(\mathcal{F}, \mathcal{S}^{\prime}, \mathcal{D}^{\prime}\right)$. Thus, there is a minimal derivation in $\left(\mathcal{F}, \mathcal{S}^{\prime}, \mathcal{D}^{\prime}\right)$ for $\operatorname{Head}(r))$ using $r$ as a last rule: $\langle d,(x, r)\rangle$ s.t. $x=\operatorname{Head}(r), \operatorname{Def}(d) \subseteq \mathcal{D}^{\prime}$ and Strict $(d) \subseteq \mathcal{S}^{\prime}$. Thus, $a=(\langle d,(x, r)\rangle, x)$ is an argument outside $\mathcal{E}$. a does not attack any argument of $\mathcal{E}$. Indeed, if we suppose the contrary then, since $\mathcal{E}$ is a preferred extension, there is $b \in \mathcal{E}$ s.t. $b \mathcal{R}_{u} a$. So, there is a sub-argument of a: $a^{\prime}=\left(\left\langle d^{\prime},\left(x^{\prime}, r^{\prime}\right)\right\rangle, x^{\prime}\right)$ with $x^{\prime} \in \mathcal{D}^{\prime}$ and $b=\left(d^{\prime \prime}, x^{\prime}\right)$. However since $a^{\prime} \in \mathcal{E}$ (because it uses only rules from $\left.S^{\prime} \cup D^{\prime}\right)$, this means that $\mathcal{E}$ is not conflict-free which contradicts the fact that $\mathcal{E}$ is a preferred extension. So $\mathcal{E} \cup\{a\}$ is conflict free. Moreover, for every $c \in \operatorname{Arg}(\mathcal{T}) \backslash(\mathcal{E} \cup\{a\})$, if $c \mathcal{R}_{u} a$ then there is a subargument of a: $a^{\prime}=\left(\left\langle d^{\prime},\left(x^{\prime}, r^{\prime}\right)\right\rangle, x^{\prime}\right)$ with $x^{\prime} \in \mathcal{D}^{\prime}$ and $c=\left(d^{\prime \prime}, x^{\prime}\right)$. However since $a^{\prime} \in \mathcal{E}$ (because it uses only rules from $S^{\prime} \cup D^{\prime}$ ) and $\mathcal{E}$ is a preferred extension, then there is $a^{\prime} \in \mathcal{E}$ such that $a^{\prime} \mathcal{R}_{u} c$. This means that $\mathcal{E} \cup\{a\}$ is conflict-free and defends all its elements. Contradiction with the fact that $\mathcal{E}$ is maximal. Now let us prove that $\mathcal{O}=\left(\mathcal{F}, \mathcal{S}, \mathcal{D}^{\prime}\right) \in \operatorname{POpt}(\mathcal{T})$.

- It is obvious that $\mathcal{D}^{\prime} \subseteq \mathcal{D}$

- Similar to the proof of point 2 in Theroem 3.

- Similar to the proof of point 3 in Theroem 3.

- $\forall \mathcal{D}^{\prime \prime} \subseteq \mathcal{D}$, if $\exists r^{\prime} \in \mathcal{D}^{\prime}$ s.t. $r^{\prime} \in \operatorname{CN}\left(\mathcal{F}, \mathcal{S}, \mathcal{D}^{\prime \prime}\right)$ then there is a minimal derivation $d^{\prime}$ for $r^{\prime}$ in $\left(\mathcal{F}, \mathcal{S}, \mathcal{D}^{\prime \prime}\right)$, i.e., $\left(d^{\prime}, r^{\prime}\right)$ is an $\operatorname{argument}$ of $\operatorname{Arg}(\mathcal{T})$. Since $r^{\prime} \in \mathcal{D}^{\prime}$, there in an argument $a=(d, x) \in \mathcal{E}$ s.t. $r^{\prime} \in \operatorname{Def}(d)$ and we have $b \mathcal{R}_{a} a$. Since $\mathcal{E}$ is a preferred extension, there is an argument $c=\left(d^{\prime \prime}, x^{\prime \prime}\right) \in \mathcal{E}$ s.t. $c \mathcal{R}_{u} b$, i.e., there is a derivation $d^{\prime \prime}$ for $r^{\prime \prime}$ in $\left(\mathcal{F}, \mathcal{S}, \mathcal{D}^{\prime}\right)$ s.t. $d^{\prime \prime} \in \operatorname{Def}\left(d^{\prime}\right)$. This means that $r^{\prime \prime} \in \operatorname{CN}\left(\mathcal{F}, \mathcal{S}, \mathcal{D}^{\prime}\right)$ and $r^{\prime \prime} \in \mathcal{D}^{\prime \prime}$.

- Suppose that there is $D^{\prime \prime}$ s.t. $D^{\prime} \subset \mathcal{D}^{\prime \prime}$ and $\mathcal{D}^{\prime \prime}$ satisfies the previous conditions. Let $\mathcal{O}^{\prime}=\left(\mathcal{F}, \mathcal{S}, \mathcal{D}^{\prime \prime}\right)$ and $\mathcal{E}^{\prime}=\operatorname{Arg}\left(\mathcal{O}^{\prime}\right)$. The conflict-freeness of $\mathcal{E}^{\prime}$ follows from the fact that $\mathcal{O}^{\prime}$ is coherent. Let $b=(d, x)$ be an argument of 
$\operatorname{Arg}(\mathcal{T}) \backslash \mathcal{E}^{\prime}$ s.t. there is an argument $a=\left(d^{\prime}, x^{\prime}\right) \in \mathcal{E}^{\prime}$ and $b \mathcal{R}_{u} a$. Thus, $x \in \operatorname{CN}(\mathcal{F}, \mathcal{S}, \operatorname{Def}(d))$ and $x \in \operatorname{Def}\left(d^{\prime}\right)$, i.e. $x \in \mathcal{D}^{\prime \prime}$. But, from the fourth condition of preferred options, there is $r^{\prime \prime} \in \operatorname{Def}(d)$ such that $r^{\prime \prime} \in \operatorname{CN}(\mathcal{O})$. So, there is an argument $a^{\prime} \in \mathcal{E}^{\prime}$ such that $a^{\prime} \mathcal{R}_{u} b$. Consequently, $\mathcal{E}^{\prime}$ is a preferred extension and $\mathcal{E} \subset \mathcal{E}^{\prime}$ which contradicts the fact that $\mathcal{E}$ is a preferred extension.

- We show by a similar way as in the second point of Theorem 5 that: for all $\mathcal{E}, \mathcal{E}^{\prime} \in$ $\operatorname{Ext}_{p}(\mathcal{H})$ if POption $(\mathcal{E})=\operatorname{POption}\left(\mathcal{E}^{\prime}\right), \mathcal{E}=\mathcal{E}^{\prime}$.

- Let $\mathcal{E} \in \operatorname{Ext}_{p}(\mathcal{H})$. Since $\operatorname{Th}(\mathcal{E}) \sqsubseteq \operatorname{POption}(\mathcal{E})$ and from the definition of functions $\operatorname{Th}$ and $\operatorname{Arg}$ it is obvious that $\mathcal{E} \subseteq \operatorname{Arg}(\operatorname{POption}(\mathcal{E}))$. Now let $a=(d, x) \in$ $\operatorname{Arg}(\operatorname{POption}(\mathcal{E})) . a=(d, x)$ is constructed from POption $(\mathcal{E})$. So, $\operatorname{Def}(d) \subseteq$ $\operatorname{Def} \mathrm{s}(\operatorname{POption}(\mathcal{E}))$. Suppose that $a \notin \mathcal{E}$. Since $\mathcal{E}$ is a preferred extension then we have two cases. The first case is that there is $b=\left(d^{\prime}, x^{\prime}\right) \in \mathcal{E}$ such that $b \mathcal{R}_{u} a$. From $b \in \mathcal{E}$ we easily deduce that $x^{\prime} \in \operatorname{CN}(\operatorname{POption}(\mathcal{E}))$. But then, from $b \mathcal{R}_{u} a$, $\operatorname{POption}(\mathcal{E})$ must be incoherent. Contradiction with the fact that $\operatorname{POption}(\mathcal{E})$ is a preferred option. The second case is that $\mathcal{E}$ does not attack $a$ but it does not defend it: there is $b=\left(d^{\prime}, x^{\prime}\right) \notin \mathcal{E}$ such that $b \mathcal{R}_{u} a$ and $\mathcal{E}$ does not attack $b$. From $b \mathcal{R}_{u} a$ we have $x^{\prime} \in d$. Since $\operatorname{Def}(d) \subseteq \operatorname{Defs}(\operatorname{POption}(\mathcal{E}))$ then $x \subseteq \operatorname{Defs}(\operatorname{POption}(\mathcal{E}))$. So, $x$ is used in at least an argument $c=\left(d^{\prime \prime}, x^{\prime \prime}\right)$ of $\mathcal{E}$ i.e., $x \in d^{\prime \prime}$. Thus, $c$ is attacked by $b$. But since $\mathcal{E}$ is a preferred extension, then it must contain an argument which attacks $b$. This contradict the hypothesis that $\mathcal{E}$ does not attack $b$.

Proof. of Theorem 8. Let $\mathcal{H}=\left(\operatorname{Arg}(\mathcal{T}), \mathcal{R}_{u}\right)$ s.t. $\operatorname{Ext}_{s}(\mathcal{H}) \neq \emptyset$.

- Let $\mathcal{O}=\left(\mathcal{F}, \mathcal{S}, \mathcal{D}^{\prime}\right) \in \operatorname{POpt}(\mathcal{T})$ and let $\mathcal{E}=\operatorname{Arg}(\mathcal{O})$. We prove that $\mathcal{E}$ is conflictfree, $\forall b \in \operatorname{Arg}(\mathcal{T}) \backslash \mathcal{E}$, if $\exists a \in \mathcal{E}$ s.t. $b \mathcal{R}_{u} a$ then $\exists c \in \mathcal{E}$ s.t. $c \mathcal{R}_{u} b$ and $\mathcal{E}$ is a maximal subset of $\operatorname{Arg}(\mathcal{T})$ satisfying the previous two conditions.

Suppose that there is two argument $a=(d, x)$ and $b=\left(d^{\prime}, x^{\prime}\right)$ in $\mathcal{E}$ s.t. $a \mathcal{R}_{u} b$, i.e., $x \in \operatorname{Def}\left(d^{\prime}\right)$. But since $d$ and $d^{\prime}$ are derivation schemas for $x$ and $x^{\prime}$ respectively in $\mathcal{O}$ we have: $x \in \mathrm{CN}(\mathcal{O})$ and $\operatorname{Def}\left(d^{\prime}\right) \subseteq \mathcal{D}^{\prime}$, so $x \in \mathcal{D}^{\prime}$. Contradiction with the coherence of preferred option $\mathcal{O}$. So, $\mathcal{E}$ is conflict-free.

Now, let us show that: $\forall b \in \operatorname{Arg}(\mathcal{T}) \backslash \mathcal{E}$, if $\exists a \in \mathcal{E}$ s.t. $b \mathcal{R}_{u} a$ then $\exists c \in \mathcal{E}$ s.t. $c \mathcal{R}_{u} b$. Let $b=(d, x) \in \operatorname{Arg}(\mathcal{T}) \backslash \mathcal{E}$ and let $a=\left(d^{\prime}, x^{\prime}\right) \in \mathcal{E}$ s.t. $b \mathcal{R}_{u} a$, i.e., $x \in \operatorname{CN}(F, S, \operatorname{Def}(d))$ and $x \in \operatorname{Def}\left(d^{\prime}\right)$. From the fourth conditions of the definition of a preferred option, there is $r^{\prime \prime} \in \operatorname{Def}(d)$ s.t. $r^{\prime \prime} \in \operatorname{CN}\left(\mathcal{F}, \mathcal{S}, \mathcal{D}^{\prime}\right)$. So, there is an argument $c=\left(d^{\prime \prime}, r^{\prime \prime}\right)$ with $d^{\prime \prime}$ a minimal derivation of $r^{\prime \prime}$ in $\mathcal{O}$. Clearly, $c \mathcal{R}_{u} b$. Finally, Suppose that $\mathcal{E}$ is not maximal w.r.t. previous conditions. Thus, there is $\mathcal{E}^{\prime}$ s.t. $\mathcal{E} \subset \mathcal{E}^{\prime}$ and $\mathcal{E}^{\prime}$ is preferred, i.e., $\mathcal{E}^{\prime}$ is an maximal conflict-free set of arguments that defends all its elements. Let $\mathcal{O}^{\prime}\left(\mathcal{F}, \mathcal{S}, \mathcal{D}^{\prime \prime}\right)=\operatorname{POption}\left(\mathcal{E}^{\prime}\right)$. Clearly, $\mathcal{D}^{\prime} \neq \mathcal{D}$, because there every argument in $\mathcal{E}^{\prime} \backslash \mathcal{E}$ uses at least a rule which is not in $\mathcal{D}^{\prime}$. Since $\mathcal{O}^{\prime}$ is a preferred option (Theorem 7), $D^{\prime \prime}$ is maximal, so $\mathcal{D}^{\prime} \subset \mathcal{D}^{\prime \prime}$. This contradicts the fact that $\mathcal{O}$ is a preferred option.

- Similar to the proof of point 2 of Theorem 6.

- Similar to the proof of point 3 of Theorem 6 .

Proof. of Corollary 6. Follows immediately from the bijection between preferred options and preferred extensions(theorems 7 - 8). 
Proof. of Corollary 7. Follows immediately from the bijection between preferred options and preferred extensions(theorems 7 - 8). 